\title{
A FREE BOUNDARY PROBLEM DESCRIBING THE SATURATED-UNSATURATED FLOW IN A POROUS MEDIUM. PART II. EXISTENCE OF THE FREE BOUNDARY IN THE 3D CASE
}

\author{
GABRIELA MARINOSCHI
}

Received 20 October 2004

We present an extension of the results given in the first part of this paper (2004) referring to the existence in the 3D case of a free boundary between the saturated and unsaturated domains that may be evidenced during the water flow into a porous medium.

\section{Review of the main results}

During a rainfall water infiltration into an unsaturated soil, zones of saturation may be developed anywhere within the flow domain. Consequently, a natural question arises: under which conditions depending on the rate at which rain water is supplied, the initial moisture distribution in the soil, the presence of underground sources and the boundary permeability, the flow domain may be separated into two parts, one saturated and the other unsaturated? Related to that, when saturation may be observed first at the ground surface? If this happens, then beginning with the time at which the soil surface reaches saturation, the so-called saturation time, a waterfront starts to move downwards and this represents the unknown interface between the saturated and unsaturated flow regimes. Situations under question have been experimentally put in evidence and various studies focusing especially on the determination of an approximate analytical solution have been done in the 1D case, for a special hydraulic model introduced in [6]. Besides it, we may cite, for example, $[5,7]$.

In a recent paper dealing with the study of a rainfall infiltration problem (see [4]) the main feature of the model focuses on a switching boundary condition on the ground surface, that is, changing the type at the moment when this one reaches saturation.

In this paper, we present a functional approach to a rainfall infiltration, find sufficient conditions under which the saturation may be first generated at the soil surface and prove in fact the existence of the free boundary in the 3D case. We mention that the hydraulic model for which we develop the theory obeys the particularities of the most used hydraulic models in soil sciences, covering a wide range of soil types, that is, those of Broadbridge-White (see [6]) and van Genuchten (see [10]).

The paper extends in fact a previous study on this subject, that is [9] which was conceived in four main parts. 
(I) Starting from the Richards' equation written in pressure form, in the first part a model that covers both the unsaturated and saturated flow particularities in a porous medium was introduced, by defining a specific multivalued function acting in the corresponding diffusive form of the model.

(II) The existence and uniqueness of the solution to the saturated-unsaturated flow model written for diffusive form of Richards' equation was proved in the three dimensional case, on the basis of the proof of similar facts for the solution of a certain approximating problem.

(III) According to some supplementary results concerning the regularity of the approximating solution, the existence of the free boundary was proved only in the one-dimensional case.

(IV) Next, the existence of the weak solution defined for the model written in pressure form was proved in the $3 \mathrm{D}$ case.

The existence of the free boundary and the uniqueness of the weak and smooth solution remained as open problems in the $3 \mathrm{D}$ case. This paper has as main scope to give an answer to these problems.

The plan of the paper includes:

(1) A summary of the diffusive model and of the main results given in [9]. A detailed study of the boundedness of the solution and its implications upon the reliability of the solution.

(2) The proof of a better regularity of the approximating solution and consequences upon the solution to the original problem. The proof of the vertical monotonicity of the solution that represents the basic result that enhances the delimitation of the flow domain into two parts, one saturated and the other unsaturated and the definition of the free boundary that separates them.

(3) A final discussion on the model in pressure and the proof of the uniqueness of its solution in the $3 \mathrm{D}$ case.

However, for a more precise understanding of the model, some details of it will be presented in Appendix A.1.

1.1. The mathematical model. Let $\Omega$ be an open bounded subset of $\mathbb{R}^{N}(N=1,2,3)$ with the boundary $\partial \Omega \stackrel{\text { notation }}{=} \Gamma$ piecewise smooth, let $(0, T)$ be a finite time interval and let $x \in \Omega$ represent the vector $x=\left(x_{1}, x_{2}, x_{3}\right)$.

We consider $\Omega$ to be the cylinder $\Omega=\left\{x ;\left(x_{1}, x_{2}\right) \in D, 0<x_{3}<L\right\}$ where $D$ is an open bounded subset of $\mathbb{R}^{N-1}$ with smooth boundary and we assume that $\Gamma$ is composed of the disjoint boundaries $\Gamma_{u}, \Gamma_{\text {lat }}$ and $\Gamma_{b}$, all sufficiently smooth where $\Gamma_{u}=\left\{x \in \Gamma ; x_{3}=0\right\}$, $\Gamma_{b}=\left\{x \in \Gamma ; x_{3}=L\right\}, \Gamma=\Gamma_{u} \cup \Gamma_{\text {lat }} \cup \bar{\Gamma}_{b}$. We also denote $\Gamma_{\alpha}=\Gamma_{\text {lat }} \cup \bar{\Gamma}_{b}$, with $\Gamma_{u} \cap \Gamma_{\alpha}=\varnothing$.

We will deal with the diffusive form of the mathematical model of a rainfall water infiltration into an isotropic, homogeneous, porous soil with the boundary $\Gamma_{\alpha}$ semipermeable

$$
\begin{gathered}
\frac{\partial \theta}{\partial t}-\Delta \beta^{*}(\theta)+\frac{\partial K(\theta)}{\partial x_{3}}=f \quad \text { in } Q=\Omega \times(0, T), \\
\theta(x, 0)=\theta_{0}(x) \quad \text { in } \Omega, \\
\left(K(\theta) i_{3}-\nabla \beta^{*}(\theta)\right) \cdot v=u \quad \text { on } \Sigma_{u}=\Gamma_{u} \times(0, T), \\
\left(K(\theta) i_{3}-\nabla \beta^{*}(\theta)\right) \cdot v=\alpha \beta^{*}(\theta)+f_{0} \quad \text { on } \Sigma_{\alpha}=\Gamma_{\alpha} \times(0, T) .
\end{gathered}
$$


In this model $v$ is the outward normal to $\Gamma, i_{3}$ is the unit vector along $O x_{3}$, downwards directed, $f$ is some source in $Q, f_{0}$ and $u$ are known on $\Sigma_{\alpha}$ and on $\Sigma_{u}$, respectively, and $K$ and $\beta^{*}$ are defined as follows (see Appendix A.1):

$$
K(\theta):=\left\{\begin{array}{ll}
0, & \theta \leq 0 \\
K(\theta), & 0<\theta \leq \theta_{s},
\end{array} \quad \beta^{*}(\theta):= \begin{cases}\rho \theta, & \theta \leq 0 \\
\int_{0}^{\theta} \beta(\xi) d \xi, & 0<\theta<\theta_{s} \\
{\left[K_{s}^{*},+\infty\right),} & \theta=\theta_{s},\end{cases}\right.
$$

where

$$
\beta(\theta)= \begin{cases}\rho, & \theta \leq 0 \\ \beta(\theta), & 0<\theta<\theta_{s},\end{cases}
$$

$K_{s}^{*}=\lim _{\theta>\theta_{s}} \beta^{*}(\theta), 0<K_{s}^{*}<\infty$ and $0<\rho<\infty$.

We consider as basic assumptions for the functions occurring in this model the following:

(a) $\alpha: \Gamma_{\alpha} \rightarrow\left[\alpha_{m}, \alpha_{M}\right]$ is positive and continuous;

(b) $K \in C^{2}\left(\left[0, \theta_{s}\right]\right)$, it is positive, monotonically increasing and convex, $K(0)=0$ and $K\left(\theta_{s}\right)=K_{s}$

(c) $\beta \in C^{2}\left(\left(0, \theta_{s}\right)\right)$, it is positive, monotonically increasing and convex and

$$
\beta(0)=\rho, \quad \lim _{\theta>\theta_{s}} \beta(\theta)=+\infty, \quad \int_{0}^{\theta_{s}} \beta(\xi) d \xi<\infty .
$$

Moreover, they satisfy

(i) $\left(\beta^{*}(\theta)-\beta^{*}(\bar{\theta})\right)(\theta-\bar{\theta}) \geq \rho(\theta-\bar{\theta})^{2}, \forall \theta, \bar{\theta} \in\left(-\infty, \theta_{s}\right]$,

(ii) $\lim _{\theta \rightarrow-\infty} \beta^{*}(\theta)=-\infty$,

(ii $\left.{ }_{\mathrm{K}}\right)|K(\theta)-K(\bar{\theta})| \leq M|\theta-\bar{\theta}|, \forall \theta, \bar{\theta} \leq \theta_{s}$.

A review of the model is presented in Appendix A.1.

1.2. Functional framework. For the sake of simplicity we will denote the scalar product and the norm in $L^{2}(\Omega)$ by $(\cdot, \cdot)$ and $\|\cdot\|$ respectively. Also we will no longer write the function arguments which represent the integration variables.

The problem was treated within the functional framework represented by $V=H^{1}(\Omega)$, with the norm defined by

$$
\|\psi\|_{V}=\left(\int_{\Omega}|\nabla \psi|^{2} d x+\int_{\Gamma_{\alpha}} \alpha(x)|\psi|^{2} d \sigma\right)^{1 / 2},
$$

$V^{\prime}=\left(H^{1}(\Omega)\right)^{\prime}$ is its dual endowed with the scalar product

$$
\langle\theta, \bar{\theta}\rangle_{V^{\prime}}=(\theta, \psi), \quad \forall \theta, \bar{\theta} \in V^{\prime},
$$


816 A free boundary problem

where $\psi \in V$ satisfies the boundary value problem

$$
-\Delta \psi=\bar{\theta}, \quad \frac{\partial \psi}{\partial \nu}+\alpha \psi=0 \quad \text { on } \Gamma_{\alpha}, \quad \frac{\partial \psi}{\partial \nu}=0 \quad \text { on } \Gamma_{u},
$$

$\left(\partial / \partial \nu\right.$ is the normal derivative). Here $(\theta, \psi)$ represents the value of $\theta \in V^{\prime}$ at $\psi \in V$, or the pairing between $V^{\prime}$ and $V$.

We set

$$
D(A)=\left\{\theta \in L^{2}(\Omega) ; \exists \eta \in V, \eta(x) \in \beta^{*}(\theta(x)) \text { a.e. } x \in \Omega\right\}
$$

and we defined the multivalued operator $A: D(A) \subset V^{\prime} \rightarrow V^{\prime}$, by

$$
(A \theta, \psi)=\int_{\Omega}\left(\nabla \eta \cdot \nabla \psi-K(\theta) \frac{\partial \psi}{\partial x_{3}}\right) d x+\int_{\Gamma_{\alpha}} \alpha \eta \psi d \sigma, \quad \forall \psi \in V .
$$

Moreover, we defined $B \in L\left(L^{2}\left(\Gamma_{u}\right) ; V^{\prime}\right)$ and $f_{\Gamma} \in L^{2}\left(0, T ; V^{\prime}\right)$ by

$$
\begin{gathered}
B u(\psi)=-\int_{\Gamma_{u}} u \psi d \sigma, \quad \forall \psi \in V, \\
f_{\Gamma}(t)(\psi)=-\int_{\Gamma_{\alpha}} f_{0} \psi d \sigma, \quad \forall \psi \in V
\end{gathered}
$$

and with these notations we introduced the Cauchy problem

$$
\begin{gathered}
\frac{d \theta}{d t}+A \theta \ni f+B u+f_{\Gamma} \quad \text { a.e. } t \in(0, T), \\
\theta(0)=\theta_{0}(x) \quad \text { in } \Omega,
\end{gathered}
$$

whose strong solution, if exists, satisfies (1.1)-(1.4) in the sense of distributions.

In order to prove the existence results the multivalued function $\beta^{*}$ was approximated by the continuous function defined for each $\varepsilon>0$ by

$$
\beta_{\varepsilon}^{*}(\theta)= \begin{cases}\beta^{*}(\theta), & \theta<\theta_{s} \\ K_{s}^{*}+\frac{\theta-\theta_{s}}{\varepsilon}, & \theta \geq \theta_{s}\end{cases}
$$


so that, besides the properties (i) (for $\theta \in \mathbb{R}$ ), (ii), $\beta_{\varepsilon}^{*}(\theta)$ satisfies also

(iii) $\lim _{\theta \rightarrow \infty} \beta_{\varepsilon}^{*}(\theta)=+\infty$.

Also in the approximating problem we extended $K$ to the right of the saturation value by the constant value $K_{s}$.

Consequently, the associated approximating problem

$$
\begin{gathered}
\frac{d \theta_{\varepsilon}}{d t}+A_{\varepsilon} \theta_{\varepsilon}=f+B u+f_{\Gamma} \quad \text { a.e. } t \in(0, T), \\
\theta_{\varepsilon}(0)=\theta_{0}(x) \quad \text { in } \Omega,
\end{gathered}
$$

followed, where $A_{\varepsilon}: D\left(A_{\varepsilon}\right) \subset V^{\prime} \rightarrow V^{\prime}$ is the single-valued operator defined by

$$
\left(A_{\varepsilon} \theta, \psi\right)=\int_{\Omega}\left(\nabla \beta_{\varepsilon}^{*}(\theta) \cdot \nabla \psi-K(\theta) \frac{\partial \psi}{\partial x_{3}}\right) d x+\int_{\Gamma_{\alpha}} \alpha \beta_{\varepsilon}^{*}(\theta) \psi d \sigma, \quad \forall \psi \in V
$$

with the domain

$$
D\left(A_{\varepsilon}\right)=\left\{\theta \in L^{2}(\Omega) ; \beta_{\varepsilon}^{*}(\theta) \in V\right\} .
$$

Obviously the strong solution to (1.17)-(1.18) is the solution in the sense of distributions to the boundary value problem

$$
\begin{gathered}
\frac{\partial \theta_{\varepsilon}}{\partial t}-\Delta \beta_{\varepsilon}^{*}\left(\theta_{\varepsilon}\right)+\frac{\partial K\left(\theta_{\varepsilon}\right)}{\partial x_{3}}=f \quad \text { in } Q, \\
\theta_{\varepsilon}(x, 0)=\theta_{0}(x) \quad \text { in } \Omega, \\
\left(K\left(\theta_{\varepsilon}\right) i_{3}-\nabla \beta_{\varepsilon}^{*}\left(\theta_{\varepsilon}\right)\right) \cdot v=u \text { on } \Sigma_{u}, \\
\left(K\left(\theta_{\varepsilon}\right) i_{3}-\nabla \beta^{*}\left(\theta_{\varepsilon}\right)\right) \cdot v=\alpha \beta_{\varepsilon}^{*}\left(\theta_{\varepsilon}\right)+f_{0} \text { on } \Sigma_{\alpha} .
\end{gathered}
$$

1.3. Existence and uniqueness of the solution. The proof of the existence of the solution to problem (1.17)-(1.18) was based on the quasi $m$-accretivity of the operator $A_{\varepsilon}$. Using an intermediate result (see [9, Proposition 4.2], see also [3]) we proved the following.

Theorem 1.1 (existence of the approximating solution). Let

$$
\begin{gathered}
f \in L^{2}\left(0, T ; V^{\prime}\right), \quad u \in L^{2}\left(\Sigma_{u}\right), \quad f_{0} \in L^{2}\left(\Sigma_{\alpha}\right), \\
\theta_{0} \in L^{2}(\Omega) ; \quad \theta_{0} \leq \theta_{s} \text { a.e. on } \Omega .
\end{gathered}
$$


818 A free boundary problem

Then, problem (1.17)-(1.18) has, for each $\varepsilon>0$, a unique strong solution

$$
\begin{gathered}
\theta_{\varepsilon} \in L^{2}(0, T ; V) \cap W^{1,2}\left(0, T ; V^{\prime}\right), \\
\beta_{\varepsilon}^{*}(\theta) \in L^{2}(0, T ; V),
\end{gathered}
$$

that satisfies the estimates

$$
\begin{gathered}
\left\|\theta_{\varepsilon}(t)\right\|_{V^{\prime}}^{2}+\int_{0}^{t}\left\|\theta_{\varepsilon}(\tau)\right\|^{2} d \tau \\
\leq \gamma_{1}\left(\alpha_{m}\right)\left(\left\|\theta_{0}\right\|_{V^{\prime}}^{2}+\int_{0}^{T}\|f(\tau)\|_{V^{\prime}}^{2} d \tau+\int_{0}^{T}\|u(\tau)\|_{L^{2}\left(\Gamma_{u}\right)}^{2} d \tau+\int_{0}^{T}\left\|f_{0}(\tau)\right\|_{L^{2}\left(\Gamma_{\alpha}\right)}^{2} d \tau\right) \\
\left\|\theta_{\varepsilon}(t)\right\|^{2} \leq \int_{\Omega} j_{\varepsilon}\left(\theta_{\varepsilon}(t)\right) d x+\int_{0}^{t}\left\|\frac{d \theta_{\varepsilon}}{d \tau}(\tau)\right\|_{V^{\prime}}^{2} d \tau+\int_{0}^{t}\left\|\beta_{\varepsilon}^{*}\left(\theta_{\varepsilon}(\tau)\right)\right\|_{V}^{2} d \tau \\
\leq \gamma_{2}\left(\alpha_{m}\right)\left(\int_{\Omega} j_{\mathcal{\varepsilon}}\left(\theta_{0}\right) d x+\int_{0}^{T}\|f(\tau)\|_{V^{\prime}}^{2} d \tau\right. \\
\left.\quad+\int_{0}^{T}\|u(\tau)\|_{L^{2}\left(\Gamma_{u}\right)}^{2} d \tau+\int_{0}^{T}\left\|f_{0}(\tau)\right\|_{L^{2}\left(\Gamma_{\alpha}\right)}^{2} d \tau\right)
\end{gathered}
$$

In the above estimates $\alpha_{m}=\min _{x \in \Gamma_{\alpha}} \alpha(x), \gamma_{1}\left(\alpha_{m}\right)=O\left(1 / \alpha_{m}\right), \gamma_{2}\left(\alpha_{m}\right)=O\left(1 / \alpha_{m}\right)$ as $\alpha_{m} \rightarrow 0$ and

$$
j_{\varepsilon}(r)=\int_{0}^{r} \beta_{\varepsilon}^{*}(\xi) d \xi
$$

Since the estimates (1.28) and (1.29) do not depend on $\varepsilon$, by passing to the limit as $\varepsilon \rightarrow 0$, it was proved that the approximating solution tends to the solution to the Cauchy problem (1.14)-(1.15) and the latter is also bounded by $\theta_{s}$ a.e. on $Q$.

Theorem 1.2 (existence of the original solution). Let $f, u, f_{0}$ and $\theta_{0}$ satisfy (1.25)-(1.26). Then there exists a unique solution $\theta \in C\left([0, T] ; L^{2}(\Omega)\right)$ to the exact problem (1.14)-(1.15) such that

$$
\begin{gathered}
\theta \in L^{2}(0, T ; V) \cap W^{1,2}\left(0, T ; V^{\prime}\right), \quad \beta^{*}(\theta) \in L^{2}(0, T ; V), \\
\theta \leq \theta_{s} \quad \text { a.e. in } Q .
\end{gathered}
$$


Moreover,

$$
\begin{gathered}
\|\theta(t)\|_{V^{\prime}}^{2}+\int_{0}^{t}\|\theta(\tau)\|^{2} d \tau \\
\leq \gamma_{1}\left(\alpha_{m}\right)\left(\left\|\theta_{0}\right\|_{V^{\prime}}^{2}+\int_{0}^{T}\|f(\tau)\|_{V^{\prime}}^{2} d \tau\right. \\
\left.\quad+\int_{0}^{T}\|u(\tau)\|_{L^{2}\left(\Gamma_{u}\right)}^{2} d \tau+\int_{0}^{T}\left\|f_{0}(\tau)\right\|_{L^{2}\left(\Gamma_{\alpha}\right)}^{2} d \tau\right), \\
\|\theta(t)\|^{2} \leq \int_{\Omega} j(\theta(t)) d x+\int_{0}^{t}\left\|\frac{d \theta}{d \tau}(\tau)\right\|_{V^{\prime}}^{2} d \tau+\int_{0}^{t}\|\eta(\tau)\|_{V^{2}}^{2} d \tau \\
\leq \gamma_{2}\left(\alpha_{m}\right)\left(\int_{\Omega}^{j} j\left(\theta_{0}\right) d x+\int_{0}^{T}\|f(\tau)\|_{V^{\prime}}^{2} d \tau\right. \\
\left.\quad+\int_{0}^{T}\|u(\tau)\|_{L^{2}\left(\Gamma_{u}\right)}^{2} d \tau+\int_{0}^{T}\left\|f_{0}(\tau)\right\|_{L^{2}\left(\Gamma_{\alpha}\right)}^{2} d \tau\right),
\end{gathered}
$$

where $\eta \in \beta^{*}(\theta)$ a.e. on $Q$ and $j: \mathbb{R} \rightarrow(-\infty, \infty]$ is defined by

$$
j(r)= \begin{cases}\int_{0}^{r} \beta^{*}(\xi) d \xi, & r \leq \theta_{s} \\ +\infty, & r>\theta_{s},\end{cases}
$$

(here, $\left.\lim _{\xi>\theta_{s}} \beta^{*}\left(\theta_{s}\right)=K_{s}^{*}\right)$.

1.4. Boundedness of the solution. A result which refers to the boundedness of the approximating solution is proved below and this will be used to show that under certain hypotheses the solution $\theta$ to the original problem (1.14)-(1.15) belongs to the physical domain for the moisture.

Let us choose two time dependent functions $\theta_{M} \in C^{1}[0, T]$ and $\theta_{m} \in C^{1}[0, T]$ such that

$$
\theta_{m}(t) \leq \theta_{M}(t), \quad \theta_{m}^{\prime}(t) \leq \theta_{M}^{\prime}(t), \quad \forall t \in[0, T]
$$

Moreover we assume that $\theta_{m}(0)$ and $\theta_{M}(0)$ do not vanish simultaneously and the same thing is true for $\theta_{m}^{\prime}(0)$ and $\theta_{M}^{\prime}(0)$.

Then, let us denote

$$
\begin{aligned}
f_{M}(t) & =\theta_{M}^{\prime}(t), \quad u_{M}(t)=-K\left(\theta_{M}(t)\right), \\
f_{0 \varepsilon}^{M}(x, t) & =K\left(\theta_{M}(t)\right) i_{3} \cdot v-\alpha(x) \beta_{\varepsilon}^{*}\left(\theta_{M}(t)\right), \\
f_{m}(t) & =\theta_{m}^{\prime}(t), \quad u_{m}(t)=-K\left(\theta_{m}(t)\right), \\
f_{0 \varepsilon}^{m}(x, t) & =K\left(\theta_{m}(t)\right) i_{3} \cdot v-\alpha(x) \beta_{\varepsilon}^{*}\left(\theta_{m}(t)\right) .
\end{aligned}
$$


It is obvious that $\theta_{M}(t)$ is the classical solution to (1.1)-(1.4) in which $f_{M}, u_{M}, f_{0 \varepsilon}^{M}$ stand for $f, u, f_{0}$, that is,

$$
\begin{gathered}
\frac{\partial \theta_{M}}{\partial t}-\Delta \beta_{\varepsilon}^{*}\left(\theta_{M}\right)+\frac{\partial K\left(\theta_{M}\right)}{\partial x_{3}}=f_{M}(t) \quad \text { in } Q, \\
\theta_{M}(x, 0)=\theta_{M}(0) \quad \text { in } \Omega, \\
\left(K\left(\theta_{M}\right) i_{3}-\nabla \beta_{\varepsilon}^{*}\left(\theta_{M}\right)\right) \cdot v=u_{M}(t) \quad \text { on } \Sigma_{u}, \\
\left(K\left(\theta_{M}\right) i_{3}-\nabla \beta_{\varepsilon}^{*}\left(\theta_{M}\right)\right) \cdot v=\alpha \beta_{\varepsilon}^{*}\left(\theta_{M}\right)+f_{0 \varepsilon}^{M}(x, t) \quad \text { on } \Sigma_{\alpha} .
\end{gathered}
$$

Analogously, $\theta_{m}(t)$ is the classical solution to (1.1)-(1.4) corresponding to $f_{m}, u_{m}, f_{0 \varepsilon}^{m}$ instead of $f, u, f_{0}$.

Lemma 1.3 (boundedness of the approximating solution). Let

$$
\begin{gathered}
f \in L^{2}\left(0, T ; V^{\prime}\right), \quad u \in L^{2}\left(\Sigma_{u}\right), \quad f_{0} \in L^{2}\left(\Sigma_{\alpha}\right), \\
\theta_{0} \in L^{2}(\Omega) ; \quad \theta_{0} \leq \theta_{s} \text { a.e. on } \Omega
\end{gathered}
$$

hold and assume still that

$$
\begin{aligned}
\theta_{m}(0) & \leq \theta_{0}(x) \leq \theta_{M}(0) \leq \theta_{s} \quad \text { a.e. in } \Omega, \\
\theta_{m}^{\prime}(t) & \leq f(x, t) \leq \theta_{M}^{\prime}(t) \quad \text { a.e. in } Q, \\
u_{M}(t) & \leq u(x, t) \leq u_{m}(t) \quad \text { a.e. on } \Sigma_{u}, \\
f_{0 \varepsilon}^{M}(x, t) & \leq f_{0}(x, t) \leq f_{0 \varepsilon}^{m}(x, t) \quad \text { a.e. on } \Sigma_{\alpha} .
\end{aligned}
$$

Then, for each $\varepsilon>0$, we have

$$
\theta_{m}(t) \leq \theta_{\varepsilon}(x, t) \leq \theta_{M}(t) \quad \text { a.e. in } \Omega \text {, for each } t \in[0, T]
$$

Proof. First of all we have to notice that the combination between the assumptions (1.39)(1.40) and (1.41)-(1.44) turns out in the assumption of the boundedness of all initial and boundary data of the problem. For example $f \in L^{2}\left(0, T ; V^{\prime}\right)$ and relationships (1.42) should be considered in the sense of distributions, but because $f$ is bounded from both sides, then it is essentially bounded, that is,

$$
f \in L^{\infty}(Q)
$$

The boundedness of the other functions

$$
u \in L^{\infty}\left(\Sigma_{u}\right), \quad f_{0} \in L^{\infty}\left(\Sigma_{\alpha}\right)
$$

is obvious. Hence, further we will replace the assumptions (1.39) by (1.46)-(1.47).

By Theorem 1.1 problem (1.17)-(1.18) has a unique solution

$$
\theta_{\varepsilon} \in W^{1,2}\left(0, T ; V^{\prime}\right) \cap L^{2}(0, T ; V) .
$$


We multiply the equation

$$
\frac{\partial\left(\theta_{\varepsilon}-\theta_{M}\right)}{\partial t}-\Delta\left(\beta_{\varepsilon}^{*}\left(\theta_{\varepsilon}\right)-\beta_{\varepsilon}^{*}\left(\theta_{M}\right)\right)+\frac{\partial K\left(\theta_{\varepsilon}\right)}{\partial x_{3}}-\frac{\partial K\left(\theta_{M}\right)}{\partial x_{3}}=f-f_{M}
$$

by $\left(\theta_{\varepsilon}(x, t)-\theta_{M}(t)\right)^{+}$and then we integrate it over $\Omega \times(0, t)$. We get

$$
\begin{aligned}
& \int_{0}^{t} \int_{\Omega}\left\{\frac{1}{2} \frac{\partial}{\partial \tau}\left[\left(\theta_{\varepsilon}-\theta_{M}\right)^{+}\right]^{2}+\nabla\left(\beta_{\varepsilon}^{*}\left(\theta_{\varepsilon}\right)-\beta_{\varepsilon}^{*}\left(\theta_{M}\right)\right) \cdot \nabla\left(\theta_{\varepsilon}-\theta_{M}\right)^{+}\right\} d x d \tau \\
& \quad+\int_{0}^{t} \int_{\Gamma_{\alpha}} \alpha\left(\beta_{\varepsilon}^{*}\left(\theta_{\varepsilon}\right)-\beta_{\varepsilon}^{*}\left(\theta_{M}\right)\right)\left(\theta_{\varepsilon}-\theta_{M}\right)^{+} d \sigma d \tau \\
& =\int_{0}^{t} \int_{\Omega}\left(K\left(\theta_{\varepsilon}\right)-K\left(\theta_{M}\right)\right) \frac{\partial\left(\theta_{\varepsilon}-\theta_{M}\right)^{+}}{\partial x_{3}} d x d \tau \\
& \quad+\int_{0}^{t} \int_{\Omega}\left(f-f_{M}\right)\left(\theta_{\varepsilon}-\theta_{M}\right)^{+} d x d \tau-\int_{0}^{t} \int_{\Gamma_{\alpha}}\left(f_{0}-f_{0 \varepsilon}^{M}\right)\left(\theta_{\varepsilon}-\theta_{M}\right)^{+} d \sigma d \tau \\
& \quad-\int_{0}^{t} \int_{\Gamma_{u}}\left(u-u_{M}\right)\left(\theta_{\varepsilon}-\theta_{M}\right)^{+} d \sigma d \tau .
\end{aligned}
$$

But

$$
\alpha\left(\beta_{\varepsilon}^{*}\left(\theta_{\varepsilon}\right)-\beta_{\varepsilon}^{*}\left(\theta_{M}\right)\right)\left(\theta_{\varepsilon}-\theta_{M}\right)^{+} \geq \alpha \rho\left(\left(\theta_{\varepsilon}-\theta_{M}\right)^{+}\right)^{2}
$$

and by Stampacchia lemma we have that

$$
\begin{aligned}
\nabla \beta_{\varepsilon}^{*}\left(\theta_{\varepsilon}\right) \cdot \nabla\left(\theta_{\varepsilon}-\theta_{M}\right)^{+} & =\beta_{\varepsilon}\left(\theta_{\varepsilon}\right) \nabla\left(\theta_{\varepsilon}-\theta_{M}\right) \cdot \nabla\left(\theta_{\varepsilon}-\theta_{M}\right)^{+} \\
& \geq \rho\left|\nabla\left(\theta_{\varepsilon}-\theta_{M}\right)^{+}\right|^{2}
\end{aligned}
$$

It follows that

$$
\begin{aligned}
& \frac{1}{2} \int_{\Omega}\left[\left(\theta_{\varepsilon}(t)-\theta_{M}(t)\right)^{+}\right]^{2} d x+\rho \int_{0}^{t}\left\|\left(\theta_{\varepsilon}(\tau)-\theta_{M}(\tau)\right)^{+}\right\|_{V}^{2} d \tau \\
& \leq \frac{1}{2} \int_{\Omega}\left[\left(\theta_{0}-\theta_{M}(0)\right)^{+}\right]^{2} d x+\int_{0}^{t} M\left\|\theta_{\varepsilon}(\tau)-\theta_{M}(\tau)\right\| \\
& \quad \times\left\|\left(\theta_{\varepsilon}(\tau)-\theta_{M}(\tau)\right)^{+}\right\|_{V} d \tau+\int_{0}^{t} \int_{\Omega}\left(f-f_{M}\right)\left(\theta_{\varepsilon}-\theta_{M}\right)^{+} d x d \tau \\
& \quad-\int_{0}^{t} \int_{\Gamma_{\alpha}}\left(f_{0}-f_{0 \varepsilon}^{M}\right)\left(\theta_{\varepsilon}-\theta_{M}\right)^{+} d \sigma d \tau-\int_{0}^{t} \int_{\Gamma_{u}}\left(u-u_{M}\right)\left(\theta_{\varepsilon}-\theta_{M}\right)^{+} d \sigma d \tau .
\end{aligned}
$$

Using the assumptions $\theta_{0}(x) \leq \theta_{M}(0)$ a.e. in $\Omega, f \leq \theta_{M}^{\prime}(t),-u(x, t) \leq K\left(\theta_{M}(t)\right)$ a.e. on $\Sigma_{u}$ and $f_{0}(x, t) \geq f_{0 \varepsilon}^{M}(x, t)$ a.e. on $\Sigma_{\alpha}$ we obtain that

$$
\begin{gathered}
\left\|\left(\theta_{\varepsilon}(t)-\theta_{M}(t)\right)^{+}\right\|^{2}+\rho \int_{0}^{t}\left\|\left(\theta_{\varepsilon}(\tau)-\theta_{M}(\tau)\right)^{+}\right\|_{V}^{2} d \tau \\
\leq \frac{M^{2}}{\rho} \int_{0}^{t}\left\|\left(\theta_{\varepsilon}(\tau)-\theta_{M}(\tau)\right)^{+}\right\|^{2} d \tau,
\end{gathered}
$$


hence by Gronwall lemma, we get that $\left\|\left(\theta_{\varepsilon}(t)-\theta_{M}(t)\right)^{+}\right\|^{2}=0$, which implies that $\theta_{\varepsilon}(x, t)$ $\leq \theta_{M}(t)$ a.e. on $\Omega$, for each $t \in[0, T]$.

Similarly, by showing that $\left\|\left(\theta_{\varepsilon}(t)-\theta_{m}(t)\right)^{-}\right\|^{2}=0$ one can obtain the lower boundedness (see [9]).

We notice that in the previous result $f_{0 \varepsilon}^{M}$ and $f_{0 \varepsilon}^{m}$ depend on $\varepsilon$. However, for a particular choice of $\theta_{m}$ and $\theta_{M}$, sufficient conditions of boundedness that do not depend on $\varepsilon$ may be found in

Corollary 1.4. Let $\theta_{m}, \theta_{M} \in C^{1}([0, T])$ satisfying

$$
\theta_{m}(t)<\theta_{s}, \quad \forall t \in[0, T], \quad \theta_{M}(t) \geq \theta_{s}, \quad \forall t \in[0, T], \theta_{M}(0)=\theta_{s} .
$$

Assume (1.46)-(1.47), (1.41)-(1.43) and

$$
K_{s}-\alpha K_{s}^{*} \leq f_{0}(x, t) \leq K\left(\theta_{m}(t)\right)-\alpha \beta^{*}\left(\theta_{m}(t)\right) \quad \text { a.e. on } \Sigma_{\alpha} .
$$

Then

$$
\theta_{m}(t) \leq \theta_{\varepsilon}(x, t) \leq \theta_{M}(t) \quad \text { a.e. in } \Omega \text {, for each } t \in[0, T] .
$$

Proof. The hypothesis $\theta_{m}(t)<\theta_{s}, \forall t \in[0, T]$, implies that $\beta_{\varepsilon}^{*}\left(\theta_{m}\right)=\beta^{*}\left(\theta_{m}\right)<\beta^{*}\left(\theta_{s}\right)$, for any $\varepsilon<d\left(\theta_{m}(t), \theta_{s}\right)$, where $d\left(\theta_{m}(t), \theta_{s}\right)=\min _{t \in[0, T]}\left(\theta_{s}-\theta_{m}(t)\right)$. Hence for $\varepsilon$ small enough the term $K\left(\theta_{m}\right)-\alpha \beta_{\varepsilon}^{*}\left(\theta_{m}\right)$ may be replaced by $K\left(\theta_{m}\right)-\alpha \beta^{*}\left(\theta_{m}\right)$ so that $f_{0 \varepsilon}^{m}$ turns out to be independent on $\varepsilon$. In particular $\theta_{m}$ can be chosen a constant less than $\theta_{s}$.

Now, for $\theta_{M}(t) \geq \theta_{s}$ we have $\beta_{\varepsilon}^{*}\left(\theta_{M}\right) \geq K_{s}^{*}$, so that

$$
K\left(\theta_{M}\right)-\alpha \beta_{\varepsilon}^{*}\left(\theta_{M}\right) \leq K_{s}-\alpha K_{s}^{*} .
$$

In conclusion, using assumption (1.56) we can write a.e. on $\Sigma_{\alpha}$ that

$$
K\left(\theta_{M}\right)-\alpha \beta_{\varepsilon}^{*}\left(\theta_{M}\right) \leq K_{s}-\alpha K_{s}^{*} \leq f_{0}(x, t) \leq K\left(\theta_{m}\right)-\alpha \beta^{*}\left(\theta_{m}\right) .
$$

The latter, together with (1.41)-(1.43) implies the boundedness of $\theta_{\varepsilon}$ between $\theta_{m}(t)$ and $\theta_{M}(t)$.

Remark 1.5. It is obvious that if, in Corollary 1.4, we choose both functions $\theta_{m}(t)$ and $\theta_{M}(t)$ less than $\theta_{s}$, it would follow a criterion of comparison only for the unsaturated case. That is why, in order to study the saturated-unsaturated flow the choice of $\theta_{M}(t) \geq \theta_{s}$ is essential.

On the other hand, as smaller is $\theta_{m}$ the larger is the interval of boundedness for $\theta$.

Corollary 1.6 (boundedness of the original solution). Assume (1.46), (1.47) and $\theta_{M}(t)$ $\geq \theta_{s}, \forall t \in(0, T]$

$$
\begin{gathered}
0 \leq \theta_{0}(x) \leq \theta_{M}(0)=\theta_{s} \quad \text { a.e. in } \Omega, \\
0 \leq f(x, t) \leq \theta_{M}^{\prime}(t) \quad \text { a.e. in } Q, \\
0 \leq-u(x, t) \leq K\left(\theta_{M}(t)\right) \quad \text { a.e. on } \Sigma_{u}, \\
K_{s}-\alpha K_{s}^{*} \leq f_{0}(x, t) \leq 0 \quad \text { a.e. on } \Sigma_{\alpha} .
\end{gathered}
$$


Then

$$
0 \leq \theta(x, t) \leq \theta_{s} \quad \text { a.e. in } \Omega, \text { for each } t \in[0, T]
$$

Proof. The proof follows immediately from Theorem 1.2 and Lemma 1.3, choosing $\theta_{m}(t)=0$ and $\theta_{M}(t)$ a non-constant function with $\theta_{M}(0) \neq 0$.

\section{Existence of the free boundary in the $3 \mathrm{D}$ case}

2.1. Supplementary regularity of the approximating solution. The proof of the existence of the free boundary requires some stronger regularity of the approximating solution. In this subsection, we will prove successively in Theorems 2.1 and 2.5 further regularity properties for the solution $\theta_{\varepsilon}$ to the approximating problem (1.17)-(1.18). They may be obtained using a smoother approximation $\beta_{\varepsilon}^{*}$ of class $C^{3}(\mathbb{R})$, so, we provide these results considering the smoother approximation (A.14). Also, we prefer to give them in two separate theorems because the proofs are quite long and technical.

Theorem 2.1. Assume that

$$
\begin{gathered}
f \in W^{1,2}\left(0, T ; L^{2}(\Omega)\right), \\
u \in W^{1,2}\left(0, T ; L^{2}\left(\Gamma_{u}\right)\right) \cap L^{2}\left(0, T ; H^{1}\left(\Gamma_{u}\right)\right), \\
f_{0} \in W^{1,2}\left(0, T ; L^{2}\left(\Gamma_{\alpha}\right)\right) \cap L^{2}\left(0, T ; H^{1}\left(\Gamma_{\alpha}\right)\right), \\
\theta_{0} \in H^{1}(\Omega), \quad \theta_{0} \leq \theta_{\text {s }} \text { a.e. on } \Omega .
\end{gathered}
$$

Then, for each $\varepsilon>0$, the solution $\theta_{\varepsilon}$ to problem (1.17)-(1.18) satisfies

$$
\begin{gathered}
\theta_{\varepsilon} \in W^{1,2}\left(0, T ; L^{2}(\Omega)\right) \cap L^{\infty}(0, T ; V) \cap L^{2}\left(0, T ; H^{2}(\Omega)\right), \\
\beta_{\varepsilon}^{*}\left(\theta_{\varepsilon}\right) \in W^{1,2}\left(0, T ; L^{2}(\Omega)\right) \cap L^{\infty}(0, T ; V) \cap L^{2}\left(0, T ; H^{2}(\Omega)\right) .
\end{gathered}
$$

Proof. By the hypotheses (2.1)-(2.4) it follows that the approximating problem has a unique solution satisfying the conclusions of Theorem 1.1 .

Since we do not know a priori that $\partial \theta_{\varepsilon} / \partial t$ and $\partial \beta_{\varepsilon}^{*}\left(\theta_{\varepsilon}\right) / \partial t$ are in $L^{2}(\Omega)$ for $t \in[0, T]$, in a rigorous way we should perform the next calculations by replacing these derivatives by the corresponding finite differences

$$
\frac{\theta_{\varepsilon}(t+\delta)-\theta_{\varepsilon}(t)}{\delta}, \quad \frac{\beta_{\varepsilon}^{*}\left(\theta_{\varepsilon}(t+\delta)\right)-\beta_{\varepsilon}^{*}\left(\theta_{\varepsilon}(t)\right)}{\delta}
$$

which belong to the same space as $\theta_{\varepsilon}$ does. However, for the writing simplicity we will denote these differences by

$$
\frac{\partial \theta_{\varepsilon}}{\partial t}, \frac{\partial \beta_{\varepsilon}^{*}\left(\theta_{\varepsilon}\right)}{\partial t}, \text { respectively, }
$$

so in the below proof this way of writing is symbolical. 
We multiply (1.17) by $\partial \beta_{\varepsilon}^{*}\left(\theta_{\varepsilon}\right) / \partial t$ and integrate over $\Omega \times(0, t)$. We have

$$
\begin{aligned}
\int_{0}^{t} \int_{\Omega} \beta_{\varepsilon}\left(\theta_{\varepsilon}\right)\left(\frac{\partial \theta_{\varepsilon}}{\partial \tau}\right)^{2} d \tau d x+\frac{1}{2} \int_{0}^{t} \frac{\partial}{\partial \tau}\left\|\nabla \beta_{\varepsilon}^{*}\left(\theta_{\varepsilon}(\tau)\right)\right\|^{2} d \tau \\
=\int_{0}^{t} \int_{\Omega} K\left(\theta_{\varepsilon}\right) \frac{\partial}{\partial x_{3}}\left(\frac{\partial \beta_{\varepsilon}^{*}\left(\theta_{\varepsilon}\right)}{\partial \tau}\right) d \tau d x+\int_{0}^{t} \int_{\Omega} f \frac{\partial \beta_{\varepsilon}^{*}\left(\theta_{\varepsilon}\right)}{\partial \tau} d \tau d x \\
\quad-\int_{0}^{t} \int_{\Gamma_{\alpha}}\left(\alpha \beta_{\varepsilon}^{*}\left(\theta_{\varepsilon}\right)+f_{0}\right) \frac{\partial \beta_{\varepsilon}^{*}\left(\theta_{\varepsilon}\right)}{\partial \tau} d \sigma d \tau-\int_{0}^{t} \int_{\Gamma_{u}} u \frac{\partial \beta_{\varepsilon}^{*}\left(\theta_{\varepsilon}\right)}{\partial \tau} d \sigma d \tau .
\end{aligned}
$$

After the integration with respect to $\tau$ of the second term in the left-hand side, we obtain

$$
\begin{aligned}
\int_{0}^{t} \int_{\Omega} \beta_{\varepsilon}\left(\theta_{\varepsilon}\right)\left(\frac{\partial \theta_{\varepsilon}}{\partial \tau}\right)^{2} d \tau d x+\frac{1}{2} \int_{\Omega}\left|\nabla \beta_{\varepsilon}^{*}\left(\theta_{\varepsilon}(t)\right)\right|^{2} d x-\frac{1}{2} \int_{\Omega}\left|\nabla \beta_{\varepsilon}^{*}\left(\theta_{0}\right)\right|^{2} d x \\
=\int_{\Omega}\left(K\left(\theta_{\varepsilon}(t)\right) \frac{\partial \beta_{\varepsilon}^{*}\left(\theta_{\varepsilon}(t)\right)}{\partial x_{3}}-K\left(\theta_{0}\right) \frac{\partial \beta_{\varepsilon}^{*}\left(\theta_{0}\right)}{\partial x_{3}}\right) d x \\
\quad-\int_{0}^{t} \int_{\Omega} \frac{\partial K\left(\theta_{\varepsilon}\right)}{\partial \tau} \frac{\partial \beta_{\varepsilon}^{*}\left(\theta_{\varepsilon}\right)}{\partial x_{3}} d x d \tau-\frac{1}{2} \int_{\Gamma_{\alpha}} \alpha\left(\left(\beta_{\varepsilon}^{*}\left(\theta_{\varepsilon}(t)\right)\right)^{2}-\left(\beta_{\varepsilon}^{*}\left(\theta_{0}\right)\right)^{2}\right) d \sigma \\
\quad+\int_{\Omega} f(t) \beta_{\varepsilon}^{*}\left(\theta_{\varepsilon}(t)\right) d x-\int_{\Omega} f(0) \beta_{\varepsilon}^{*}\left(\theta_{0}\right) d x-\int_{0}^{t} \int_{\Omega} \frac{\partial f}{\partial \tau} \beta_{\varepsilon}^{*}\left(\theta_{\varepsilon}\right) d x d \tau \\
\quad-\int_{\Gamma_{\alpha}} f_{0}(t) \beta_{\varepsilon}^{*}\left(\theta_{\varepsilon}(t)\right) d \sigma+\int_{\Gamma_{\alpha}} f_{0}(0) \beta_{\varepsilon}^{*}\left(\theta_{0}\right) d \sigma+\int_{0}^{t} \int_{\Gamma_{\alpha}} \frac{\partial f_{0}}{\partial \tau} \beta_{\varepsilon}^{*}\left(\theta_{\varepsilon}\right) d \sigma d \tau \\
\quad-\int_{\Gamma_{u}} u(t) \beta_{\varepsilon}^{*}\left(\theta_{\varepsilon}(t)\right) d \sigma+\int_{\Gamma_{u}} u(0) \beta_{\varepsilon}^{*}\left(\theta_{0}\right) d \sigma+\int_{0}^{t} \int_{\Gamma_{u}} \frac{\partial u}{\partial \tau} \beta_{\varepsilon}^{*}\left(\theta_{\varepsilon}\right) d \sigma d \tau .
\end{aligned}
$$

Now, by trace theorem and Poincaré's inequality it follows that there exists $c_{V H}, c_{H}$, $c_{\Gamma_{u}}$, and $c_{\Gamma_{\alpha}}$ such that $\forall \psi \in H^{1}(\Omega)$ we have

$$
\begin{gathered}
\|\psi\|_{V} \leq c_{V H}\|\psi\|_{H^{1}(\Omega)}, \quad\|\psi\|_{H^{1}(\Omega)} \leq c_{H}\|\psi\|_{V}, \\
\|\psi\|_{L^{2}\left(\Gamma_{u}\right)} \leq c_{\Gamma_{u}}\|\psi\|_{V}, \quad\|\psi\|_{L^{2}\left(\Gamma_{\alpha}\right)} \leq c_{\Gamma_{\alpha}}\|\psi\|_{V},
\end{gathered}
$$

where $c_{\Gamma_{u}}^{2}$ and $c_{\Gamma_{\alpha}}^{2}$ depend on $1 / \alpha_{m}$ (see also [3]). We remind that $\alpha_{m}>0$. Using further (2.11) which applies for $\beta_{\varepsilon}^{*}\left(\theta_{\varepsilon}\right) \in V$, we have

$$
\begin{aligned}
\int_{0}^{t} \int_{\Omega} \beta_{\varepsilon}\left(\theta_{\varepsilon}\right)\left(\frac{\partial \theta_{\varepsilon}}{\partial \tau}\right)^{2} d \tau d x+\frac{1}{2} \int_{\Omega}\left|\nabla \beta_{\varepsilon}^{*}\left(\theta_{\varepsilon}(t)\right)\right|^{2} d x+\frac{1}{2} \int_{\Gamma_{\alpha}} \alpha\left(\beta_{\varepsilon}^{*}\left(\theta_{\varepsilon}(t)\right)\right)^{2} d \sigma \\
\leq C_{0}^{\varepsilon}+\left\|K\left(\theta_{\varepsilon}(t)\right)\right\|\left\|\beta_{\varepsilon}^{*}\left(\theta_{\varepsilon}(t)\right)\right\|_{V}+M \int_{0}^{t}\left\|\frac{\partial \theta_{\varepsilon}}{\partial \tau}(\tau)\right\|\left\|\beta_{\varepsilon}^{*}\left(\theta_{\varepsilon}(\tau)\right)\right\|_{V} d \tau \\
\quad+c_{H}\|f(t)\|\left\|\beta_{\varepsilon}^{*}\left(\theta_{\varepsilon}(t)\right)\right\|_{V}+c_{H} \int_{0}^{t}\left\|\frac{\partial f}{\partial \tau}(\tau)\right\|\left\|\beta_{\varepsilon}^{*}\left(\theta_{\varepsilon}(\tau)\right)\right\|_{V} d \tau \\
\quad+\left\|f_{0}(t)\right\|_{L^{2}\left(\Gamma_{\alpha}\right)}\left\|\beta_{\varepsilon}^{*}\left(\theta_{\varepsilon}(t)\right)\right\|_{L^{2}\left(\Gamma_{\alpha}\right)}+\int_{0}^{t}\left\|\frac{\partial f_{0}}{\partial \tau}(\tau)\right\|_{L^{2}\left(\Gamma_{\alpha}\right)}\left\|\beta_{\varepsilon}^{*}\left(\theta_{\varepsilon}(\tau)\right)\right\|_{L^{2}\left(\Gamma_{\alpha}\right)} d \tau \\
\quad+\|u(t)\|_{L^{2}\left(\Gamma_{u}\right)}\left\|\beta_{\varepsilon}^{*}\left(\theta_{\varepsilon}(t)\right)\right\|_{L^{2}\left(\Gamma_{u}\right)}+\int_{0}^{t}\left\|\frac{\partial u}{\partial \tau}(\tau)\right\|_{L^{2}\left(\Gamma_{u}\right)}\left\|\beta_{\varepsilon}^{*}\left(\theta_{\varepsilon}(\tau)\right)\right\|_{L^{2}\left(\Gamma_{u}\right)} d \tau,
\end{aligned}
$$


where

$$
\begin{aligned}
C_{0}^{\varepsilon}= & \frac{1}{2} \int_{\Omega}\left|\nabla \beta_{\varepsilon}^{*}\left(\theta_{0}\right)\right|^{2} d x-\int_{\Omega} K\left(\theta_{0}\right) \frac{\partial \beta_{\varepsilon}^{*}\left(\theta_{0}\right)}{\partial x_{3}} d x-\int_{\Omega} f(0) \beta_{\varepsilon}^{*}\left(\theta_{0}\right) d x \\
& +\frac{1}{2} \int_{\Gamma_{\alpha}} \alpha\left(\beta_{\varepsilon}^{*}\left(\theta_{0}\right)\right)^{2} d \sigma+\int_{\Gamma_{\alpha}} f_{0}(0) \beta_{\varepsilon}^{*}\left(\theta_{0}\right) d \sigma+\int_{\Gamma_{u}} u(0) \beta_{\varepsilon}^{*}\left(\theta_{0}\right) d \sigma .
\end{aligned}
$$

Since $\beta_{\varepsilon}\left(\theta_{\varepsilon}\right) \geq \beta_{m}$ (see Appendix A.2, (A.19)) we can write

$$
\begin{aligned}
\beta_{m} \int_{0}^{t} \int_{\Omega}\left(\frac{\partial \theta_{\varepsilon}}{\partial \tau}\right)^{2} d \tau d x+\frac{1}{2}\left\|\beta_{\varepsilon}^{*}\left(\theta_{\varepsilon}\right)\right\|_{V}^{2} \\
\leq\left|C_{0}^{\varepsilon}\right|+\frac{\beta_{m}}{2} \int_{0}^{t} \int_{\Omega}\left(\frac{\partial \theta_{\varepsilon}}{\partial \tau}\right)^{2} d \tau d x+\frac{1}{2} \int_{0}^{t}\left(\frac{M^{2}}{\beta_{m}}+3\right)\left\|\beta_{\varepsilon}^{*}\left(\theta_{\varepsilon}(\tau)\right)\right\|_{V}^{2} d \tau \\
\quad+\frac{1}{2} \int_{0}^{t}\left(c_{H}^{2}\left\|\frac{\partial f}{\partial \tau}(\tau)\right\|^{2}+c_{\Gamma_{\alpha}}^{2}\left\|\frac{\partial f_{0}}{\partial \tau}(\tau)\right\|_{L^{2}\left(\Gamma_{\alpha}\right)}^{2}+c_{\Gamma_{u}}^{2}\left\|\frac{\partial u}{\partial \tau}(\tau)\right\|_{L^{2}\left(\Gamma_{u}\right)}^{2}\right) d \tau \\
\quad+\frac{1}{4}\left\|\beta_{\varepsilon}^{*}\left(\theta_{\varepsilon}(\tau)\right)\right\|_{V}^{2}+4 c_{H}^{2}\|f(t)\|^{2}+4 c_{\Gamma_{\alpha}}^{2}\left\|f_{0}(t)\right\|_{L^{2}\left(\Gamma_{\alpha}\right)}^{2}+4 c_{\Gamma_{u}}^{2}\|u(t)\|_{L^{2}\left(\Gamma_{u}\right)}^{2} \\
\quad+4 M^{2}\left\|\theta_{\varepsilon}(t)\right\|^{2} .
\end{aligned}
$$

Here we used (2.12) and $\left(\mathrm{i}_{\mathrm{K}}\right)$. Taking into account the hypotheses and the estimate $\left\|\theta_{\varepsilon}\right\|^{2} \leq C_{0}$, (where $C_{0}$ is the $\varepsilon$-independent right-hand side sum in (1.29)), we get

$$
\begin{aligned}
& \frac{\beta_{m}}{2} \int_{0}^{t} \int_{\Omega}\left(\frac{\partial \theta_{\varepsilon}}{\partial \tau}\right)^{2} d \tau d x+\frac{1}{4}\left\|\beta_{\varepsilon}^{*}\left(\theta_{\varepsilon}(t)\right)\right\|_{V}^{2} \\
& \quad \leq\left|C_{0}^{\varepsilon}\right|+C_{1}+C_{2}+C_{3} \int_{0}^{t}\left\|\beta_{\varepsilon}^{*}\left(\theta_{\varepsilon}(\tau)\right)\right\|_{V}^{2} d \tau
\end{aligned}
$$

where

$$
\begin{aligned}
& C_{1}=\frac{1}{2}\left(c_{H}^{2}\left\|\frac{\partial f}{\partial t}\right\|_{L^{2}(Q)}^{2}+c_{\Gamma_{\alpha}}^{2}\left\|\frac{\partial f_{0}}{\partial t}\right\|_{L^{2}\left(\Sigma_{\alpha}\right)}^{2}+c_{\Gamma_{u}}^{2}\left\|\frac{\partial u}{\partial t}\right\|_{L^{2}\left(\Sigma_{u}\right)}^{2}\right), \\
& C_{2}=4\left\{c_{H}^{2}\|f\|_{L^{\infty}\left(0, T ; L^{2}(\Omega)\right)}^{2}+c_{\Gamma_{\alpha}}^{2}\left\|f_{0}\right\|_{L^{\infty}\left(0, T ; L^{2}\left(\Sigma_{\alpha}\right)\right)}^{2}+c_{\Gamma_{u}}^{2}\|u\|_{L^{\infty}\left(0, T ; L^{2}\left(\Sigma_{u}\right)\right)}^{2}+M^{2} C_{0}^{2}\right\}, \\
& C_{3}=\frac{1}{2}\left(\frac{M^{2}}{\beta_{m}}+3\right) .
\end{aligned}
$$

Here, we noticed that the assumptions (2.1)-(2.3) imply $f \in C\left([0, T] ; L^{2}(\Omega)\right) \subset L^{\infty}(0, T$; $\left.L^{2}(\Omega)\right), u \in L^{\infty}\left(0, T ; L^{2}\left(\Gamma_{u}\right)\right)$ and $f_{0} \in L^{\infty}\left(0, T ; L^{2}\left(\Gamma_{\alpha}\right)\right)$.

Using Gronwall's lemma we finally obtain that

$$
\left\|\beta_{\varepsilon}^{*}\left(\theta_{\varepsilon}(t)\right)\right\|_{V}^{2} \leq 4\left(\left|C_{0}^{\varepsilon}\right|+C_{1}+C_{2}\right) e^{4 C_{3} t}, \quad \forall t \in[0, T]
$$

hence

$$
\left\|\beta_{\varepsilon}^{*}\left(\theta_{\varepsilon}(t)\right)\right\|_{V}^{2} \leq C_{1}^{\varepsilon}, \quad \forall t \in[0, T]
$$


that is, $\beta_{\varepsilon}^{*}\left(\theta_{\varepsilon}\right) \in L^{\infty}(0, T ; V)$ and

$$
\left\|\frac{d \theta_{\varepsilon}}{d t}\right\|_{L^{2}(Q)} \leq C_{2}^{\varepsilon},
$$

where

$$
C_{1}^{\varepsilon}=4\left(\left|C_{0}^{\varepsilon}\right|+C_{1}+C_{2}\right) e^{4 C_{3} T}, \quad C_{2}^{\varepsilon}=\frac{2}{\beta_{m}}\left(\left|C_{0}^{\varepsilon}\right|+C_{1}+C_{2}+C_{3} \cdot C_{1}^{\varepsilon} T\right) .
$$

The condition (i) implies that the function $\left(\beta_{\varepsilon}^{*}\right)^{-1}: \mathbb{R} \rightarrow \mathbb{R}$ is Lipschitz, so from (2.19) we also obtain that

$$
\left\|\theta_{\varepsilon}(t)\right\|_{V}^{2} \leq C(\varepsilon), \quad \forall t \in[0, T] .
$$

From now on, within this proof, we will denote by $C(\varepsilon)$ some constants that depend on $\varepsilon$ (by the means of $\left|C_{0}^{\varepsilon}\right|$ ).

We also derive that $K\left(\theta_{\varepsilon}\right) \in L^{\infty}\left(0, T ; H^{1}(\Omega)\right)$ and therefore we deduce that $\left.K\left(\theta_{\varepsilon}\right)\right|_{\Sigma} \in$ $L^{\infty}\left(0, T ; H^{1 / 2}(\Gamma)\right)$, (see, e.g., [8]).

Now we resume (1.17) to get

$$
\Delta \beta_{\varepsilon}^{*}\left(\theta_{\varepsilon}\right)=\frac{\partial \theta_{\varepsilon}}{\partial t}-\frac{\partial K\left(\theta_{\varepsilon}\right)}{\partial x_{3}}-f \in L^{2}\left(0, T ; L^{2}(\Omega)\right),
$$

which yields

$$
\left\|\Delta \beta_{\varepsilon}^{*}\left(\theta_{\varepsilon}\right)\right\|_{L^{2}(Q)} \leq C(\varepsilon) .
$$

By the boundary conditions (1.23), (1.24) and the hypotheses we may deduce that

$$
\begin{gathered}
\left.\nabla \beta_{\varepsilon}^{*}\left(\theta_{\varepsilon}\right) \cdot v\right|_{\Sigma_{u}}=\left.\left(K\left(\theta_{\varepsilon}\right) i_{3} \cdot v-u\right)\right|_{\Sigma_{u}} \in L^{2}\left(0, T ; H^{1 / 2}\left(\Gamma_{u}\right)\right), \\
\left.\left(\nabla \beta_{\varepsilon}^{*}\left(\theta_{\varepsilon}\right) \cdot v+\alpha \beta_{\varepsilon}^{*}\left(\theta_{\varepsilon}\right)\right)\right|_{\Sigma_{\alpha}}=\left.\left(K\left(\theta_{\varepsilon}\right) i_{3} \cdot v-f_{0}\right)\right|_{\Sigma_{\alpha}} \in L^{2}\left(0, T ; H^{1 / 2}\left(\Gamma_{\alpha}\right)\right)
\end{gathered}
$$

which together with (2.24) implies in virtue of the trace theorem (see [8]) that

$$
\beta_{\varepsilon}^{*}\left(\theta_{\varepsilon}\right) \in L^{2}\left(0, T ; H^{2}(\Omega)\right)
$$

and therefore

$$
\theta_{\varepsilon} \in L^{2}\left(0, T ; H^{2}(\Omega)\right)
$$

The last assertion is proved by noticing that by (2.26)

$$
\begin{gathered}
g=\frac{\partial \beta_{\varepsilon}^{*}\left(\theta_{\varepsilon}\right)}{\partial x_{i}} \in L^{2}\left(0, T ; H^{1}(\Omega)\right), \quad \frac{\partial \theta_{\varepsilon}}{\partial x_{i}}=\frac{g}{\beta_{\varepsilon}\left(\theta_{\varepsilon}\right)}, \\
\frac{\partial}{\partial x_{j}} \frac{\partial \theta_{\varepsilon}}{\partial x_{i}}=\frac{g_{x_{j}}}{\beta_{\varepsilon}\left(\theta_{\varepsilon}\right)}-\frac{\beta_{\varepsilon}^{\prime}\left(\theta_{\varepsilon}\right)}{\beta_{\varepsilon}^{2}\left(\theta_{\varepsilon}\right)} g \frac{\partial \theta_{\varepsilon}}{\partial x_{j}},
\end{gathered}
$$

where $g_{x_{j}} \in L^{2}\left(0, T ; L^{2}(\Omega)\right)$ and $\beta_{\varepsilon}\left(\theta_{\varepsilon}\right) \geq \beta_{m}>0$ on $Q$. 
Now, by the Sobolev embedding theorem (see, e.g., [2]) we have for any $\eta \in H^{1}(\Omega) \subset$ $L^{6}(\Omega)$ that

$$
\int_{\Omega} \eta^{4} d x \leq\left(\int_{\Omega} \eta^{2} d x\right)^{1 / 2}\left(\int_{\Omega} \eta^{6} d x\right)^{1 / 2}=\|\eta\|\|\eta\|_{L^{6}(\Omega)}^{3}
$$

and therefore

$$
\int_{\Omega} \eta^{4} d x \leq C_{4}\|\eta\|\|\eta\|_{H^{1}(\Omega)}^{3}, \quad \forall \eta \in H^{1}(\Omega)
$$

(where we denoted by $C_{4}$ the constant occurring in the Sobolev embedding formula). Therefore

$$
\begin{aligned}
\left\|g \frac{\partial \theta_{\varepsilon}}{\partial x_{j}}\right\|_{L^{2}(Q)}^{2} & =\int_{0}^{T} \int_{\Omega} g^{2}\left(\frac{\partial \theta_{\varepsilon}}{\partial x_{j}}\right)^{2} d x d t \\
& \leq \int_{0}^{T}\left(\int_{\Omega} g^{4} d x\right)^{1 / 2}\left(\int_{\Omega}\left(\frac{\partial \theta_{\varepsilon}}{\partial x_{j}}\right)^{6} d x\right)^{1 / 2} d t \\
& \leq \int_{0}^{T} C_{4}^{2}\|g(t)\|^{1 / 2}\|g(t)\|_{H^{1}(\Omega)}^{3 / 2}\left\|\frac{\partial \theta_{\varepsilon}}{\partial x_{j}}(t)\right\|^{1 / 2}\left\|\frac{\partial \theta_{\varepsilon}}{\partial x_{j}}(t)\right\|_{H^{1}(\Omega)}^{3 / 2} d t .
\end{aligned}
$$

Recalling (2.28) and (2.29) we obtain that

$$
\int_{0}^{T}\left\|\frac{\partial \theta_{\varepsilon}}{\partial x_{j}}(t)\right\|_{H^{1}(\Omega)}^{2} d t \leq C(\varepsilon)\left(1+\int_{0}^{T}\left\|\frac{\partial \theta_{\varepsilon}}{\partial x_{j}}(t)\right\|^{2} d t\right)+\frac{1}{2} \int_{0}^{T}\left\|\frac{\partial \theta_{\varepsilon}}{\partial x_{j}}\right\|_{H^{1}(\Omega)}^{2} d t
$$

implying finally that

$$
\int_{0}^{T}\left\|\frac{\partial \theta_{\varepsilon}(t)}{\partial x_{j}}\right\|_{H^{1}(\Omega)}^{2} d t \leq C(\varepsilon)
$$

By all these, together with the conclusions of Theorem 1.1 we get (2.5)-(2.6) as claimed.

Remark 2.2. In this proof $C_{0}, C_{1}, C_{2}, C_{3}, C_{4}$ are some constants independent on $\varepsilon$ and $C_{0}^{\varepsilon}, C_{1}^{\varepsilon}, C_{2}^{\varepsilon}$ and $C(\varepsilon)$ are constants depending on $\varepsilon$, so the result is true only for each $\varepsilon>0$ apart.

Therefore, in the case when $\theta_{0} \leq \theta_{s}$ a.e. on $\Omega$, we can show a better regularity only for the approximating solution $\theta_{\varepsilon}$, but we can not pass to limit to get a similar result for $\theta$.

However, under a particular assumption we may deduce a further regularity for $\theta$ too.

Theorem 2.3. Under hypotheses (2.1)-(2.3) and

$$
\theta_{0} \in H^{1}(\Omega), \quad \text { ess } \sup _{x \in \Omega} \theta_{0} \leq \theta_{s}-\delta,
$$


the solution $\theta$ to problem (1.14) satisfies in addition

$$
\begin{aligned}
\theta & \in W^{1,2}\left(0, T ; L^{2}(\Omega)\right) \cap L^{\infty}(0, T ; V) \cap L^{2}\left(0, T ; H^{2}(\Omega)\right), \\
\beta^{*}(\theta) & \in W^{1,2}\left(0, T ; L^{2}(\Omega)\right) \cap L^{\infty}(0, T ; V) \cap L^{2}\left(0, T ; H^{2}(\Omega)\right) .
\end{aligned}
$$

Proof. Since $\theta_{0} \in H^{1}(\Omega)$, ess $\sup _{x \in \Omega} \theta_{0}<\theta_{s}$, it follows that we may write $\theta_{0} \leq \operatorname{ess~sup}_{x \in \Omega} \theta_{0}$ $\leq \theta_{s}-d\left(\theta_{0}, \theta_{s}\right)$, where $d\left(\theta_{0}, \theta_{s}\right)>0$ is the fixed distance between $\theta_{0}$ and $\theta_{s}$. Then, $\beta_{\varepsilon}^{*}\left(\theta_{0}\right)=$ $\beta^{*}\left(\theta_{0}\right) \in H^{1}(\Omega)$, for any $\varepsilon<d\left(\theta_{0}, \theta_{s}\right)$. By the hypotheses (2.1)-(2.3) and Theorem 1.2 we obtain that problem (1.14)-(1.15) has a unique solution

$$
\theta \in L^{2}(0, T ; V) \cap W^{1,2}\left(0, T ; V^{\prime}\right), \quad \beta^{*}(\theta) \in L^{2}(0, T ; V), \quad \theta \leq \theta_{s} \text { a.e. in } Q .
$$

At the same time we get by Theorem 2.1 that the approximating solution to (1.17)(1.18) satisfies

$$
\begin{gathered}
\theta_{\varepsilon} \in W^{1,2}\left(0, T ; L^{2}(\Omega)\right) \cap L^{\infty}(0, T ; V) \cap L^{2}\left(0, T ; H^{2}(\Omega)\right), \\
\beta_{\varepsilon}^{*}\left(\theta_{\varepsilon}\right) \in W^{1,2}\left(0, T ; L^{2}(\Omega)\right) \cap L^{\infty}(0, T ; V) \cap L^{2}\left(0, T ; H^{2}(\Omega)\right) .
\end{gathered}
$$

But due to the hypothesis $\operatorname{essup}_{x \in \Omega} \theta_{0}<\theta_{s}$ we notice that the constant $C_{0}^{\varepsilon}$ given by (2.14) becomes independent on $\varepsilon$, since we may replace $\beta_{\varepsilon}^{*}\left(\theta_{0}\right)$ by $\beta^{*}\left(\theta_{0}\right) \in H^{1}(\Omega)$ for all $\varepsilon<\delta$.

In conclusion, at their turn, the right-hand constants in (2.19)-(2.24) and (2.34) do no longer depend on $\varepsilon$, so we may conclude that

$$
\begin{gathered}
\theta_{\varepsilon} \longrightarrow \theta \text { weakly in } L^{2}\left(0, T ; H^{2}(\Omega)\right) \text { and weak star in } L^{\infty}(0, T ; V), \\
\frac{d \theta_{\varepsilon}}{d t} \longrightarrow \frac{d \theta}{d t} \text { weakly in } L^{2}\left(0, T ; L^{2}(\Omega)\right), \\
\beta_{\varepsilon}^{*}\left(\theta_{\varepsilon}\right) \longrightarrow \beta^{*}(\theta) \text { weakly in } L^{2}\left(0, T ; H^{2}(\Omega)\right) \cap W^{1,2}\left(0, T ; L^{2}(\Omega)\right) \\
\text { and weak star in } L^{\infty}(0, T ; V) .
\end{gathered}
$$

Therefore, by trace theorem, we still obtain that

$$
\left.\left.\beta_{\varepsilon}^{*}\left(\theta_{\varepsilon}\right)\right|_{\Sigma_{\alpha}} \longrightarrow \beta^{*}(\theta)\right|_{\Sigma_{\alpha}} \text { weakly in } L^{2}\left(0, T ; H^{3 / 2}\left(\Gamma_{\alpha}\right)\right) .
$$

Finally, we get also that

$$
\theta_{\varepsilon} \longrightarrow \theta \text { strongly in } L^{2}(0, T ; V) .
$$

Hence we have proved that the solution to problem (1.14)-(1.15) belongs to the spaces indicated in (2.36).

Remark 2.4. In the special case when the initial data is less than $\theta_{s}$, we have found that

$$
\theta(t) \in H^{2}(\Omega) \quad \text { for } t \in(0, T) .
$$

This means that we get that $\theta(t) \in C(\bar{\Omega})$ for $N=1,2,3$, while in the $1 \mathrm{D}$ case (when $\left.H^{2}(\Omega) \subset C^{1}(\bar{\Omega})\right)$ the solution turns out to be differentiable continuous with respect to the spatial variable. 
We notice that in the $1 \mathrm{D}$ case, since we have that $\theta \in W^{1,2}(Q)$, it follows in addition that the solution $\theta$ is continuous with respect to both variables,

$$
\theta \in C(\bar{Q}) \quad \text { if } N=1 \text {. }
$$

Theorem 2.5. Assume that

$$
\begin{gathered}
f \in W^{1,2}\left(0, T ; L^{2}(\Omega)\right), \\
u \in W^{1,2}\left(0, T ; L^{2}\left(\Gamma_{u}\right)\right) \cap L^{2}\left(0, T ; H^{1}\left(\Gamma_{u}\right)\right), \\
f_{0} \in W^{1,2}\left(0, T ; L^{2}\left(\Gamma_{\alpha}\right)\right) \cap L^{2}\left(0, T ; H^{1}\left(\Gamma_{\alpha}\right)\right), \\
\theta_{0} \in H^{2}(\Omega), \quad \theta_{0} \leq \theta_{\text {s }} \text { a.e. on } \Omega .
\end{gathered}
$$

Then, for each $\varepsilon>0$, the problem (1.17)-(1.18) has a unique solution

$$
\begin{gathered}
\theta_{\varepsilon} \in W^{1, \infty}\left(0, T ; L^{2}(\Omega)\right) \cap W^{1,2}(0, T ; V) \cap L^{\infty}\left(0, T ; H^{2}(\Omega)\right), \\
\beta_{\varepsilon}^{*}\left(\theta_{\varepsilon}\right) \in W^{1, \infty}\left(0, T ; L^{2}(\Omega)\right) \cap W^{1,2}(0, T ; V) \cap L^{\infty}\left(0, T ; H^{2}(\Omega)\right) .
\end{gathered}
$$

Proof. By the hypotheses (2.45)-(2.48) it follows that the approximating problem has a unique solution satisfying the conclusions of Theorem 2.1.

We will show that $\beta_{\varepsilon}^{*}\left(\theta_{\varepsilon}\right) \in W^{1, \infty}\left(0, T ; L^{2}(\Omega)\right)$.

We denote

$$
\begin{gathered}
\eta=\beta_{\varepsilon}^{*}\left(\theta_{\varepsilon}\right), \quad \theta_{\varepsilon}=\left(\beta_{\varepsilon}^{*}\right)^{-1}(\eta), \quad \zeta(\eta)=K\left(\left(\beta_{\varepsilon}^{*}\right)^{-1}(\eta)\right), \\
\omega(\eta)=\frac{1}{\beta_{\varepsilon}\left(\left(\beta_{\varepsilon}^{*}\right)^{-1}(\eta)\right)}
\end{gathered}
$$

and we notice that for each $\varepsilon>0$ the functions $\beta_{\varepsilon}, \beta_{\varepsilon}^{\prime}$ and $\beta_{\varepsilon}^{\prime \prime}$ are bounded

$$
\beta_{m} \leq \beta_{\varepsilon}\left(\theta_{\varepsilon}\right) \leq \beta_{M}(\varepsilon), \quad \beta_{m}^{\prime} \leq \beta_{\varepsilon}^{\prime}\left(\theta_{\varepsilon}\right) \leq \beta_{M}^{\prime}(\varepsilon), \quad \beta_{m}^{\prime \prime} \leq \beta_{\varepsilon}^{\prime \prime}\left(\theta_{\varepsilon}\right) \leq \beta_{M}^{\prime \prime}(\varepsilon),
$$

where

$$
\begin{gathered}
\beta_{m}=\min _{\theta_{\varepsilon} \in \mathbb{R}} \beta_{\varepsilon}\left(\theta_{\varepsilon}\right), \quad \beta_{m}^{\prime}=\min _{\theta_{\varepsilon} \in \mathbb{R}} \beta_{\varepsilon}^{\prime}\left(\theta_{\varepsilon}\right), \quad \beta_{m}^{\prime \prime}=\min _{\theta_{\varepsilon} \in \mathbb{R}} \beta_{\varepsilon}^{\prime \prime}\left(\theta_{\varepsilon}\right), \\
\beta_{M}(\varepsilon)=\max _{\theta_{\varepsilon} \in \mathbb{R}} \beta_{\varepsilon}\left(\theta_{\varepsilon}\right), \quad \beta_{M}^{\prime}(\varepsilon)=\max _{\theta_{\varepsilon} \in \mathbb{R}} \beta_{\varepsilon}^{\prime}\left(\theta_{\varepsilon}\right),
\end{gathered}
$$

We still mention that $\beta_{m}, \beta_{m}^{\prime}$ and $\beta_{m}^{\prime \prime}$ do not depend on $\varepsilon$ as seen in Appendix A.2, (A.17)-(A.18), but $\beta_{M}, \beta_{M}^{\prime}, \beta_{M}^{\prime \prime}$ depend and have the order of $1 / \varepsilon$ as $\varepsilon \rightarrow 0$.

Then, we replace $\beta_{\varepsilon}^{*}\left(\theta_{\varepsilon}\right)$ by $\eta$ in (1.21)-(1.24) and, for example, (1.21) becomes

$$
\omega(\eta) \eta_{t}-\Delta \eta+\frac{\partial \zeta(\eta)}{\partial x_{3}}=f \quad \text { in } Q
$$

where $\eta_{t}$ is the derivative of $\eta$ with respect to $t$. Correspondingly we write the initial and boundary conditions in the new variable $\eta$. 
We differentiate the equations with respect to $t$ and obtain

$$
\begin{gathered}
\omega^{\prime}(\eta)\left(\eta_{t}\right)^{2}+\omega(\eta) \eta_{t t}-\Delta \eta_{t}+\frac{\partial}{x_{3}}\left(\zeta^{\prime}(\eta) \eta_{t}\right)=f_{t} \quad \text { in } Q, \\
\left(\zeta^{\prime}(\eta) \eta_{t} i_{3}-\nabla \eta_{t}\right) \cdot v=u_{t} \quad \text { on } \Sigma_{u}, \\
\left(\zeta^{\prime}(\eta) \eta_{t} i_{3}-\nabla \eta_{t}\right) \cdot v=\alpha \eta_{t}+\left(f_{0}\right)_{t} \quad \text { on } \Sigma_{\alpha}, \\
\eta_{t}(x, 0)=\eta_{t 0}(x) \quad \text { in } \Omega,
\end{gathered}
$$

where $\omega^{\prime}(\eta)$ and $\zeta^{\prime}(\eta)$ represent the derivatives of $\omega$ and $\zeta$ with respect to $\eta$, that is,

$$
\omega^{\prime}(\eta)=-\frac{1}{\beta_{\varepsilon}^{3}\left(\left(\beta_{\varepsilon}^{*}\right)^{-1}(\eta)\right)}, \quad \zeta^{\prime}(\eta)=\frac{K^{\prime}\left(\left(\beta_{\varepsilon}^{*}\right)^{-1}(\eta)\right)}{\beta_{\varepsilon}\left(\left(\beta_{\varepsilon}^{*}\right)^{-1}(\eta)\right)} \leq \frac{M}{\beta_{m}} .
$$

Equation (2.59) makes sense since $\theta_{0} \in H^{2}(\Omega)$. In fact for each $\varepsilon>0$ we have

$$
\left\|\eta_{t}(0)\right\|=\left\|\beta_{\varepsilon}\left(\theta_{0}\right)\left(\theta_{\varepsilon}\right)_{t}(0)\right\| \leq \beta_{M}(\varepsilon)\left\|\left(\theta_{\varepsilon}\right)_{t}(0)\right\|<\infty,
$$

since from (1.21) and the assumptions (2.45) and (2.48) we know that

$$
\left\|\left(\theta_{\varepsilon}\right)_{t}(0)\right\| \leq\left\|\Delta \beta_{\varepsilon}^{*}\left(\theta_{0}\right)\right\|+M\left\|\frac{\partial \theta_{0}}{\partial x_{3}}\right\|+\|f(0)\|<\infty .
$$

Then we multiply (2.56) by $\eta_{t}$ and integrate it over $\Omega \times(0, t)$. We have

$$
\begin{aligned}
& \int_{0}^{t} \int_{\Omega}\left(\omega^{\prime}(\eta) \eta_{\tau}^{3}+\frac{1}{2} \omega(\eta) \frac{\partial}{\partial \tau}\left(\eta_{\tau}^{2}\right)+\left|\nabla \eta_{\tau}\right|^{2}-\zeta^{\prime}(\eta) \eta_{\tau} \frac{\partial \eta_{\tau}}{\partial x_{3}}\right) d x d \tau \\
& \quad+\int_{0}^{t} \int_{\Gamma_{\alpha}}\left(\alpha \eta_{\tau}^{2}+\left(f_{0}\right)_{\tau}\right) \eta_{\tau} d \sigma d \tau=\int_{0}^{t} \int_{\Omega} f_{\tau} \eta_{\tau} d x d \tau+\int_{0}^{t} \int_{\Gamma_{u}} u_{\tau} \eta_{\tau} d \sigma d \tau
\end{aligned}
$$

We integrate with respect to $\tau$ the second term on the left-hand side and obtain

$$
\begin{aligned}
\frac{1}{2} \int_{\Omega} \omega( & \eta) \eta_{t}^{2}(t) d x+\int_{0}^{t}\left\|\eta_{\tau}(\tau)\right\|_{V}^{2} d \tau \\
\leq & \frac{1}{2} \int_{0}^{t} \int_{\Omega} \frac{1}{\beta_{\varepsilon}^{3}\left(\left(\beta_{\varepsilon}^{*}\right)^{-1}(\eta)\right)} \eta_{\tau}^{3} d x d \tau \\
& +\frac{1}{2} \int_{\Omega} \omega\left(\eta_{0}\right) \eta_{t}^{2}(0) d x+\int_{0}^{t} \int_{\Omega} \zeta^{\prime}(\eta) \eta_{\tau} \frac{\partial \eta_{\tau}}{\partial x_{3}} d x d \tau \\
& +\int_{0}^{t} \int_{\Omega} f_{\tau} \eta_{\tau} d x d \tau+\int_{0}^{t} \int_{\Gamma_{u}} u_{\tau} \eta_{\tau} d \sigma d \tau-\int_{0}^{t} \int_{\Gamma_{\alpha}}\left(f_{0}\right)_{\tau} \eta_{\tau} d \sigma d \tau .
\end{aligned}
$$


Taking into account (2.60) we have

$$
\begin{aligned}
& \frac{1}{2 \beta_{M}(\varepsilon)} \int_{\Omega} \eta_{t}^{2}(t) d x+\int_{0}^{t}\left\|\eta_{\tau}(\tau)\right\|_{V}^{2} d \tau \\
& \leq \frac{1}{2 \beta_{m}^{3}} \int_{0}^{t} \int_{\Omega}\left|\eta_{\tau}\right|^{3} d x d \tau+\frac{1}{2} \int_{\Omega} \omega\left(\eta_{0}\right) \eta_{t}^{2}(0) d x \\
& \quad+\frac{M}{\beta_{m}} \int_{0}^{t}\left\|\eta_{\tau}(\tau)\right\|\left\|\eta_{\tau}(\tau)\right\|_{V} d \tau+\int_{0}^{t}\left\|f_{\tau}(\tau)\right\|\left\|\eta_{\tau}(\tau)\right\| d \tau \\
& \quad+c_{\Gamma_{u}} \int_{0}^{t}\left\|u_{\tau}(\tau)\right\|_{L^{2}\left(\Gamma_{u}\right)}\left\|\eta_{\tau}(\tau)\right\|_{V} d \tau+c_{\Gamma_{\alpha}} \int_{0}^{t}\left\|\left(f_{0}\right)_{\tau}(\tau)\right\|_{L^{2}\left(\Gamma_{\alpha}\right)}\left\|\eta_{\tau}(\tau)\right\|_{V} d \tau
\end{aligned}
$$

and therefore

$$
\begin{aligned}
\frac{1}{2 \beta_{M}(\varepsilon)} \int_{\Omega} \eta_{t}^{2}(t) d x+\int_{0}^{t}\left\|\eta_{\tau}(\tau)\right\|_{V}^{2} d \tau \\
\leq \frac{1}{2 \beta_{m}^{3}} \int_{0}^{t} \int_{\Omega}\left|\eta_{\tau}\right|^{3} d x d \tau+\frac{1}{2}\left(\frac{3 M^{2}}{\beta_{m}^{2}}+1\right) \int_{0}^{t}\left\|\eta_{\tau}(\tau)\right\|^{2} d \tau \\
\quad+\frac{1}{2} \int_{0}^{t}\left\|\eta_{\tau}(\tau)\right\|_{V}^{2} d \tau+\frac{c_{1}^{\varepsilon}(t)}{2}
\end{aligned}
$$

where

$$
\begin{aligned}
c_{1}^{\varepsilon}(t)= & \int_{\Omega} \omega\left(\eta_{0}\right) \eta_{t}^{2}(0) d x+\int_{0}^{t}\left\|f_{\tau}(\tau)\right\|^{2} d \tau \\
& +3 c_{\Gamma_{u}}^{2} \int_{0}^{t}\left\|u_{\tau}(\tau)\right\|_{L^{2}\left(\Gamma_{u}\right)} d \tau+3 c_{\Gamma_{\alpha}}^{2} \int_{0}^{t}\left\|\left(f_{0}\right)_{\tau}(\tau)\right\|_{L^{2}\left(\Gamma_{\alpha}\right)} d \tau \\
\leq & \frac{1}{\beta_{m}}\left\|\eta_{t}(0)\right\|^{2}+c_{1}, \\
c_{1}= & \int_{0}^{T}\left\|f_{\tau}(\tau)\right\|^{2} d \tau+3 c_{\Gamma_{u}}^{2} \int_{0}^{T}\left\|u_{\tau}(\tau)\right\|_{L^{2}\left(\Gamma_{u}\right)} d \tau \\
& +3 c_{\Gamma_{\alpha}}^{2} \int_{0}^{T}\left\|\left(f_{0}\right)_{\tau}(\tau)\right\|_{L^{2}\left(\Gamma_{\alpha}\right)} d \tau .
\end{aligned}
$$

After a few calculations we obtain

$$
\begin{aligned}
& \frac{1}{\beta_{M}(\varepsilon)}\left\|\eta_{t}(t)\right\|^{2}+\int_{0}^{t}\left\|\eta_{\tau}(\tau)\right\|_{V}^{2} d \tau \\
& \quad \leq c_{0}^{\varepsilon}+c_{2} \int_{0}^{t}\left\|\eta_{\tau}(\tau)\right\|^{2} d \tau+c_{3} \int_{Q}\left|\eta_{\tau}\right|^{3} d x d \tau
\end{aligned}
$$

where

$$
c_{0}^{\varepsilon}=\frac{\left\|\eta_{t}(0)\right\|^{2}+\beta_{m} c_{1}}{\beta_{m}}, \quad c_{2}=\frac{3 M^{2}}{\beta_{m}^{2}}+1, \quad c_{3}=\frac{1}{\beta_{m}^{3}} .
$$


We are going to estimate now the term $\int_{\Omega}\left|\eta_{\tau}\right|^{3} d x$, using the Hölder inequality.

$$
\int_{\Omega}\left|\eta_{\tau}\right|^{3} d x=\int_{\Omega}\left|\eta_{\tau}\right|^{3 / 2}\left|\eta_{\tau}\right|^{3 / 2} d x \leq\left(\int_{\Omega}\left|\eta_{\tau}\right|^{2} d x\right)^{3 / 4}\left(\int_{\Omega}\left|\eta_{\tau}\right|^{6} d x\right)^{1 / 4}
$$

Further we can still write via Sobolev's embedding theorem

$$
\int_{\Omega}\left|\eta_{\tau}\right|^{3} d x \leq\left\|\eta_{\tau}(\tau)\right\|^{3 / 2}\left\|\eta_{\tau}(\tau)\right\|_{L^{6}(\Omega)}^{3 / 2} \leq C_{4}\left\|\eta_{\tau}(\tau)\right\|^{3 / 2}\left\|\eta_{\tau}(\tau)\right\|_{H^{1}(\Omega)}^{3 / 2}
$$

By $C_{4}$ we denoted the same constant occurring in (2.31).

We mention that by $C$ and $C(\varepsilon)$, we will further denote some constants independent and dependent on $\varepsilon$, respectively.

Therefore we have

$$
\begin{aligned}
\int_{Q}\left|\eta_{\tau}\right|^{3} d x d \tau & \leq C \int_{0}^{t}\left\|\eta_{\tau}(\tau)\right\|^{3 / 2}\left\|\eta_{\tau}(\tau)\right\|_{V}^{3 / 2} d \tau \\
& \leq C \int_{0}^{t}\left\|\eta_{\tau}(\tau)\right\|^{6} d \tau+\frac{3}{4} \int_{0}^{t}\left\|\eta_{\tau}(\tau)\right\|_{V}^{2} d \tau
\end{aligned}
$$

Here we used the inequality

$$
a b \leq \frac{a^{p}}{p}+\frac{b^{q}}{q}, \quad \frac{1}{p}+\frac{1}{q}=1
$$

with $p=4$ and $q=4 / 3$.

Inserting (2.72) in (2.68) we obtain that

$$
\frac{1}{\beta_{M}(\varepsilon)}\left\|\eta_{t}(t)\right\|^{2}+\frac{1}{4} \int_{0}^{t}\left\|\eta_{\tau}(\tau)\right\|_{V}^{2} d \tau \leq c_{0}^{\varepsilon}+C \int_{0}^{t}\left(\left\|\eta_{\tau}(\tau)\right\|^{2}+\left\|\eta_{\tau}(\tau)\right\|^{6}\right) d \tau .
$$

If we denote

$$
\varphi(t)=\left\|\eta_{t}(t)\right\|^{2} \geq 0
$$

the inequality (2.74) becomes

$$
0 \leq \frac{1}{\beta_{M}(\varepsilon)} \varphi(t) \leq c_{0}^{\varepsilon}+C \int_{0}^{t}\left(\varphi(\tau)+\varphi^{3}(\tau)\right) d \tau \leq c_{0}^{\varepsilon}+2 C \int_{0}^{t} \varphi^{3}(\tau) d \tau,
$$

if we assume that $\varphi(t) \geq 1$, in the other case $(\varphi(t)<1)$ the proof is finished, $\eta_{t}(t)$ following to be bounded in $L^{2}(\Omega)$.

Thus we obtain

$$
0 \leq \varphi(t) \leq c_{1}^{\varepsilon}+c_{2}^{\varepsilon} \int_{0}^{t} \varphi^{3}(\tau) d \tau,
$$

where $c_{1}^{\varepsilon}=c_{0}^{\varepsilon} \beta_{M}(\varepsilon), c_{2}^{\varepsilon}=2 C \beta_{M}(\varepsilon)$.

We will prove that this implies the boundedness of $\varphi(t)$. 
We define

$$
\begin{gathered}
\Phi(t)=c_{2}^{\varepsilon} \int_{0}^{t} \varphi^{3}(\tau) d \tau, \quad \Phi(0)=0, \\
\Phi^{\prime}(t)=c_{2}^{\varepsilon} \varphi^{3}(t) .
\end{gathered}
$$

Hence we obtain the differential inequality

$$
\frac{d \Phi}{\left(c_{1}^{\varepsilon}+\Phi\right)^{3}} \leq c_{2}^{\varepsilon} d t, \quad \Phi(0)=0
$$

whose solution is

$$
c_{1}^{\varepsilon}+\Phi(t) \leq \frac{c_{1}^{\varepsilon}}{\sqrt{1-2\left(c_{1}^{\varepsilon}\right)^{2} c_{2}^{\varepsilon} t}} \text { for } 0 \leq t \leq \frac{1}{2\left(c_{1}^{\varepsilon}\right)^{2} c_{2}^{\varepsilon}} .
$$

In fact we obtained

$$
0 \leq\left\|\eta_{t}(t)\right\|^{2} \leq \frac{c_{1}^{\varepsilon}}{\sqrt{1-2\left(c_{1}^{\varepsilon}\right)^{2} c_{2}^{\varepsilon} t}} \text { for } 0 \leq t<T_{0},
$$

where

$$
T_{0}=\frac{1}{2\left(c_{1}^{\varepsilon}\right)^{2} c_{2}^{\varepsilon}}
$$

If $T_{0} \geq T$ then we get from (2.74) that

$$
\eta_{t}=\frac{\partial \beta_{\varepsilon}^{*}\left(\theta_{\varepsilon}\right)}{\partial t} \in L^{\infty}\left(0, T ; L^{2}(\Omega)\right) \cap L^{2}(0, T ; V) .
$$

If $T_{0}<T$ we have the inequality (2.81) for any $t \in\left[0, t_{0}\right]$, where

$$
t_{0}=T_{0}-\delta
$$

with $\delta>0$ arbitrary and sufficiently small.

Next, we repeat the procedure for the intervals $\left[t_{0}, t_{1}\right], \ldots,\left[t_{n-1}, t_{n}\right]$ until the whole time interval $[0, T]$ is covered by $\bigcup_{j=1, \ldots, n}\left[t_{j-1}, t_{j}\right]$ where

$$
\begin{gathered}
t_{j} \in\left(T_{j}-\delta, T_{j}\right), \quad T_{j}-t_{j-1}=\frac{1}{2\left(c_{t_{j-1}}^{\varepsilon}\right)^{2} c_{2}^{\varepsilon}}, \\
c_{t_{j}}^{\varepsilon}=\beta_{M}(\varepsilon) \frac{\left\|\eta_{t}\left(t_{j-1}\right)\right\|^{2}+\beta_{m} c_{1}}{\beta_{m}} .
\end{gathered}
$$

Here a problem arises by the fact that the norm $\left\|\eta_{t}\left(t_{j-1}\right)\right\|^{2}$ may increase determining a high decrease of the time step $T_{j}-t_{j-1}$ and making thus impossible the reaching of the final time $T$. 
However we can prove that this is not the case, by using a previous estimate for $\eta_{t}(t)$, namely (2.20) from where we deduce that

$$
\int_{0}^{T}\left\|\eta_{t}(t)\right\|^{2} d t \leq \beta_{M}^{2}(\varepsilon) \int_{0}^{T}\left\|\theta_{t}(t)\right\|^{2} d t \leq \beta_{M}^{2}(\varepsilon) C_{2}^{\varepsilon}=C_{M}^{\varepsilon}
$$

which is independent of the time step (here $C_{2}^{\varepsilon}$ is that in formula (2.20)). Therefore the function $t \rightarrow\left\|\eta_{t}(t)\right\|^{2}$ belongs to $L^{2}(0, T)$, so by Luzin's theorem we have that for each $\delta>0$ there exists $E_{\delta}$ with meas $\left(E_{\delta}\right) \leq \delta / 2$ such that $\left\|\eta_{t}(t)\right\|^{2} \leq C_{M}^{\varepsilon}$ for any $t \in[0, T] \backslash E_{\delta}$. In particular the point $t$ can be found in an interval of measure $\delta$, so for example $t \in$ $(T-\delta, T)$.

Applying this result to the interval $\left(t_{j-1}, T_{j}\right)$, we can find a point $t_{j} \in\left[T_{j}-\delta, T_{j}\right)$ such that

$$
\left\|\eta_{t}\left(t_{j}\right)\right\|^{2} \leq C_{M}^{\varepsilon}
$$

and therefore

$$
\begin{aligned}
T_{j+1}-t_{j} & =\frac{1}{2\left(c_{t_{j}}^{\varepsilon}\right)^{2} c_{2}^{\varepsilon}}=\frac{1}{2 c_{2}^{\varepsilon}} \frac{1}{\beta_{M}^{2}(\varepsilon)}\left(\frac{\beta_{m}}{\left\|\eta_{t}\left(t_{j}\right)\right\|^{2}+\beta_{m} c_{1}}\right)^{2} \\
& \geq \frac{1}{4 C \beta_{M}^{3}(\varepsilon)}\left(\frac{\beta_{m}}{C_{M}^{\varepsilon}+\beta_{m} c_{1}}\right)^{2}
\end{aligned}
$$

which is independently on the time step. The procedure stops when

$$
[0, T] \subset \bigcup_{j=1, \ldots, n}\left[t_{j-1}, t_{j}\right]
$$

and resuming (2.74) we obtain thus once again (2.83), that is,

$$
\frac{\partial \beta_{\varepsilon}^{*}\left(\theta_{\varepsilon}\right)}{\partial t} \in L^{\infty}\left(0, T ; L^{2}(\Omega)\right) \cap L^{2}(0, T ; V) .
$$

Finally, taking into account (2.83) and returning to $\theta_{\varepsilon}$ we obtain (see also (2.53))

$$
\begin{gathered}
\beta_{\varepsilon}\left(\theta_{\varepsilon}\right) \frac{\partial \theta_{\varepsilon}}{\partial t} \in L^{\infty}\left(0, T ; L^{2}(\Omega)\right) \cap L^{2}(0, T ; V), \\
\frac{\partial \theta_{\varepsilon}}{\partial t} \in L^{\infty}\left(0, T ; L^{2}(\Omega)\right) \cap L^{2}(0, T ; V),
\end{gathered}
$$

and from (1.21) we deduce that

$$
\left\|\Delta \beta_{\varepsilon}^{*}\left(\theta_{\varepsilon}(t)\right)\right\| \leq\|f(t)\|+\left\|\frac{\partial \theta_{\varepsilon}}{\partial t}(t)\right\|+\left\|\frac{\partial K\left(\theta_{\varepsilon}\right)}{\partial t}(t)\right\|
$$

that is,

$$
\left\|\Delta \beta_{\varepsilon}^{*}\left(\theta_{\varepsilon}(t)\right)\right\| \leq C(\varepsilon), \quad \forall t \in[0, T]
$$


implying successively that

$$
\begin{gathered}
\beta_{\varepsilon}^{*}\left(\theta_{\varepsilon}\right) \in L^{\infty}\left(0, T ; H^{2}(\Omega)\right), \\
\nabla \beta_{\varepsilon}^{*}\left(\theta_{\varepsilon}\right)=\beta_{\varepsilon}\left(\theta_{\varepsilon}\right) \nabla \theta_{\varepsilon} \in L^{\infty}\left(0, T ; H^{1}(\Omega)\right) .
\end{gathered}
$$

Completely similar as done before (see the inequalities beginning with (2.28)) we get that

$$
\left\|\frac{\partial \theta_{\varepsilon}}{\partial x_{j}}(t)\right\|_{H^{1}(\Omega)}^{2} \leq C(\varepsilon)\left(1+\left\|g_{x_{j}}\right\|\right)+\frac{1}{2}\left\|\frac{\partial \theta_{\varepsilon}}{\partial x_{j}}(t)\right\|_{H^{1}(\Omega)}^{2}, \quad \forall t \in[0, T]
$$

so finally we obtain

$$
\theta_{\varepsilon} \in L^{\infty}\left(0, T ; H^{2}(\Omega)\right)
$$

as claimed.

Remark 2.6. However, we have to mention that some of the constants occurring in the Theorem 2.5 before depend on $\varepsilon$ by the means of $\beta_{M}(\varepsilon)$ which is unbounded if $\varepsilon \rightarrow 0$ (see especially $C_{M}^{\varepsilon}$ ). So that the estimates are true only for each $\varepsilon>0$, apart and they can not be used in order to obtain a similar regularity for the function $\theta$, neither in the case specified in Theorem 2.3.

2.2. Existence of the vertical derivative. We introduce

$$
w_{\varepsilon} \stackrel{\text { def. }}{=} \frac{\partial \theta_{\varepsilon}}{\partial x_{3}}
$$

and since $w_{\varepsilon} \in L^{2}(0, T ; V)$ by the previous theorems, we can differentiate with respect to $x_{3}$ in (1.21)-(1.24) and obtain the equivalent model for the derivative $w_{\varepsilon}$

$$
\begin{gathered}
\frac{\partial w_{\varepsilon}}{\partial t}-\Delta\left(\beta_{\varepsilon}\left(\theta_{\varepsilon}\right) w_{\varepsilon}\right)+\frac{\partial}{\partial x_{3}}\left(K^{\prime}\left(\theta_{\varepsilon}\right) w_{\varepsilon}\right)=f_{x_{3}}, \\
K\left(\theta_{\varepsilon}\right)-\beta_{\varepsilon}\left(\theta_{\varepsilon}\right) w_{\varepsilon}=-u \quad \text { on } \Sigma_{u}, \\
K\left(\theta_{\varepsilon}\right)-\beta_{\varepsilon}\left(\theta_{\varepsilon}\right) w_{\varepsilon}=\alpha \beta_{\varepsilon}^{*}\left(\theta_{\varepsilon}\right)+f_{0} \quad \text { on } \Sigma_{b}, \\
\left(K^{\prime}\left(\theta_{\varepsilon}\right) w_{\varepsilon} i_{3}-\nabla\left(\beta_{\varepsilon}\left(\theta_{\varepsilon}\right) w_{\varepsilon}\right)\right) \cdot v=\alpha \beta_{\varepsilon}\left(\theta_{\varepsilon}\right) w_{\varepsilon}+\alpha_{x_{3}} \beta_{\varepsilon}^{*}\left(\theta_{\varepsilon}\right)+\left(f_{0}\right)_{x_{3}} \quad \text { on } \Sigma_{\text {lat }},
\end{gathered}
$$

to which we add the initial condition

$$
w_{\varepsilon}(x, 0)=w_{\varepsilon 0}(x) \quad \text { in } \Omega
$$

which makes sense, due to $(2.49)\left(\partial \theta_{\varepsilon} / \partial x_{3} \in W^{1,2}\left(0, T ; L^{2}(\Omega)\right)\right)$, so $w_{\varepsilon 0} \in L^{2}(\Omega)$.

Next we will prove that if the initial and boundary data have a sufficient regularity, the problem (2.98)-(2.102) has a solution $w_{\varepsilon}$. 
Theorem 2.7. Assume that $\alpha \in C^{1}\left(\Gamma_{\alpha}\right)$, and

$$
\begin{gathered}
\theta_{0} \in H^{2}(\Omega), \quad \theta_{0} \leq \theta_{s}, \\
u \in W^{1,2}\left(0, T ; L^{2}\left(\Gamma_{u}\right)\right) \cap L^{2}\left(0, T ; H^{1}\left(\Gamma_{u}\right)\right) \cap L^{\infty}\left(\Sigma_{u}\right), \\
f_{0} \in W^{1,2}\left(0, T ; L^{2}\left(\Gamma_{\alpha}\right)\right) \cap L^{2}\left(0, T ; H^{1}\left(\Gamma_{\alpha}\right)\right) \cap L^{\infty}\left(\Sigma_{\alpha}\right), \\
f \in W^{1,2}\left(0, T ; L^{2}(\Omega)\right) .
\end{gathered}
$$

Then problem (2.98)-(2.102) has a unique solution

$$
\begin{gathered}
w_{\varepsilon} \in C\left([0, T] ; L^{2}(\Omega)\right) \cap L^{2}(0, T ; V), \\
\frac{d w_{\varepsilon}}{d t} \in L^{2}\left(0, T ; V^{\prime}\right) .
\end{gathered}
$$

Proof. By Theorem 2.5 we know that problem (1.17)-(1.18) has a unique solution

$$
\begin{aligned}
\theta_{\varepsilon} & \in W^{1, \infty}\left(0, T ; L^{2}(\Omega)\right) \cap W^{1,2}(0, T ; V) \cap L^{\infty}\left(0, T ; H^{2}(\Omega)\right), \\
\beta_{\varepsilon}^{*}\left(\theta_{\varepsilon}\right) & \in W^{1, \infty}\left(0, T ; L^{2}(\Omega)\right) \cap W^{1,2}(0, T ; V) \cap L^{\infty}\left(0, T ; H^{2}(\Omega)\right) .
\end{aligned}
$$

We introduce the functions

$$
w_{\varepsilon}^{u}=\left.\frac{K\left(\theta_{\varepsilon}\right)+u}{\beta_{\varepsilon}\left(\theta_{\varepsilon}\right)}\right|_{\Sigma_{u}}, \quad w_{\varepsilon}^{b}=\left.\frac{K\left(\theta_{\varepsilon}\right)-\alpha \beta_{\varepsilon}^{*}\left(\theta_{\varepsilon}\right)-f_{0}}{\beta_{\varepsilon}\left(\theta_{\varepsilon}\right)}\right|_{\Sigma_{b}} .
$$

They are well defined on $\Sigma_{u}$ and $\Sigma_{b}$, respectively, as we can see further. First, for any $\gamma$, $\eta \in H^{1}(\Omega)$ we have

$$
\gamma \eta \in L^{2}(\Omega)
$$

because we have by (2.31) that

$$
\begin{aligned}
\|\gamma \eta\|^{2} & =\int_{\Omega} \gamma^{2} \eta^{2} d x \leq\left(\int_{\Omega} \gamma^{4} d x\right)^{1 / 2}\left(\int_{\Omega} \eta^{4} d x\right)^{1 / 2} \\
& \leq C_{4}^{2}\|\gamma\|^{1 / 2}\|\gamma\|_{H^{1}(\Omega)}^{3 / 2}\|\eta\|^{1 / 2}\|\eta\|_{H^{1}(\Omega)}^{3 / 2}<\infty,
\end{aligned}
$$

$\left(C_{4}\right.$ is the same from $\left.(2.31)\right)$. Since $\theta_{\varepsilon}(t) \in L^{\infty}\left(0, T ; H^{2}(\Omega)\right)$ and $\beta_{\varepsilon} \in C^{2}(\mathbb{R})$ it follows that

$$
\begin{gathered}
\frac{\partial \beta_{\varepsilon}\left(\theta_{\varepsilon}\right)}{\partial x_{i}}=\beta_{\varepsilon}^{\prime}\left(\theta_{\varepsilon}\right) \frac{\partial \theta_{\varepsilon}}{\partial x_{i}} \in L^{2}\left(0, T, L^{2}(\Omega)\right), \\
\frac{\partial^{2} \beta_{\varepsilon}\left(\theta_{\varepsilon}\right)}{\partial x_{i} \partial x_{j}}=\beta_{\varepsilon}^{\prime \prime}\left(\theta_{\varepsilon}\right) \frac{\partial \theta_{\varepsilon}}{\partial x_{j}} \frac{\partial \theta_{\varepsilon}}{\partial x_{i}}+\beta_{\varepsilon}^{\prime}\left(\theta_{\varepsilon}\right) \frac{\partial^{2} \theta_{\varepsilon}}{\partial x_{i} \partial x_{j}} \in L^{2}\left(0, T ; L^{2}(\Omega)\right),
\end{gathered}
$$

the latter being implied by (2.112). We also used the fact that $\beta_{\varepsilon}$ and its derivatives up to the second order are bounded for each $\varepsilon>0$ (see Appendix A.2). In conclusion we get that

$$
\beta_{\varepsilon}\left(\theta_{\varepsilon}\right) \in L^{\infty}\left(0, T ; H^{2}(\Omega)\right),
$$


so its trace exists on $\Sigma_{u}$ and

$$
\left.\beta_{\varepsilon}\left(\theta_{\varepsilon}\right)\right|_{\Sigma_{u}} \in L^{\infty}\left(0, T ; H^{3 / 2}\left(\Gamma_{u}\right)\right)
$$

(see, e.g., [8]). Also we have that

$$
\begin{aligned}
\beta_{\varepsilon}^{\prime}\left(\theta_{\varepsilon}\right) & \in L^{\infty}\left(0, T ; H^{1}(\Omega)\right), \\
\left.\beta_{\varepsilon}^{\prime}\left(\theta_{\varepsilon}\right)\right|_{\Sigma_{u}} & \in L^{\infty}\left(0, T ; H^{1 / 2}\left(\Gamma_{u}\right)\right) .
\end{aligned}
$$

Analogously, $K \in C^{2}(\mathbb{R})$ (by regularization) and it follows that

$$
K\left(\theta_{\varepsilon}\right) \in L^{\infty}\left(0, T ; H^{2}(\Omega)\right),
$$

its trace exists on $\Sigma_{u}$ and $\left.K\left(\theta_{\varepsilon}\right)\right|_{\Sigma_{u}} \in L^{\infty}\left(0, T ; H^{3 / 2}\left(\Gamma_{u}\right)\right)$.

Hence $w_{\varepsilon}^{u}$ is well defined on $\Sigma_{u}$. Then we have to calculate

$$
\frac{\partial w_{\varepsilon}^{u}}{\partial x_{i}}=\left.\frac{\partial}{\partial x_{i}}\left(\frac{u}{\beta_{\varepsilon}\left(\theta_{\varepsilon}\right)}+\frac{K\left(\theta_{\varepsilon}\right)}{\beta_{\varepsilon}\left(\theta_{\varepsilon}\right)}\right)\right|_{\Sigma_{u}} .
$$

We will detail the explanations only for the first term, the result being the same for the second, too. Because the surface $\Gamma_{u}$ (of equation $x_{3}=0$ ) is sufficiently smooth and $u \in$ $L^{\infty}\left(\Sigma_{u}\right) \cap L^{2}\left(0, T ; H^{1}\left(\Gamma_{u}\right)\right)$, we have

$$
\left.\frac{\partial}{\partial x_{i}}\left(\frac{u}{\beta_{\varepsilon}\left(\theta_{\varepsilon}\right)}\right)\right|_{\Sigma_{u}}=\left.\left(\frac{u_{x_{i}}}{\beta_{\varepsilon}\left(\theta_{\varepsilon}\right)}-\frac{1}{\beta_{\varepsilon}^{2}\left(\theta_{\varepsilon}\right)} \frac{\partial \beta_{\varepsilon}\left(\theta_{\varepsilon}\right)}{\partial x_{i}} u\right)\right|_{\Sigma_{u}} \in L^{2}\left(\Sigma_{u}\right),
$$

so finally we get

$$
w_{\varepsilon}^{u} \in L^{2}\left(0, T ; H^{1}\left(\Gamma_{u}\right)\right)
$$

In a similar way we can show, using (2.109) and (2.110) that

$$
\frac{\partial w_{\varepsilon}^{u}}{\partial t}=\left.\frac{\partial}{\partial t}\left(\frac{u}{\beta_{\varepsilon}\left(\theta_{\varepsilon}\right)}+\frac{K\left(\theta_{\varepsilon}\right)}{\beta_{\varepsilon}\left(\theta_{\varepsilon}\right)}\right)\right|_{\Sigma_{u}} \in L^{2}\left(0, T ; L^{2}\left(\Gamma_{u}\right)\right) .
$$

Analogously it can be shown that

$$
w_{\varepsilon}^{b} \in L^{2}\left(0, T ; H^{1}\left(\Gamma_{b}\right)\right), \quad \frac{\partial w_{\varepsilon}^{b}}{\partial t} \in L^{2}\left(0, T ; L^{2}\left(\Gamma_{b}\right)\right) .
$$

By trace theorem we deduce therefore that there exists a function

$$
\widetilde{w}_{\varepsilon} \in L^{2}\left(0, T ; H^{3 / 2}(\Omega)\right) \subset L^{2}\left(0, T ; H^{1}(\Omega)\right),
$$

with

$$
\frac{\partial \tilde{w}_{\varepsilon}}{\partial t} \in L^{2}\left(0, T ; L^{2}(\Omega)\right)
$$


838 A free boundary problem

such that

$$
\left.\widetilde{w}_{\varepsilon}\right|_{\Sigma_{u}}=w_{\varepsilon}^{u},\left.\quad \widetilde{w}_{\varepsilon}\right|_{\Sigma_{b}}=w_{\varepsilon}^{b}
$$

Here is the argument. Since we may assume that $\widetilde{w}_{\mathcal{E}} \in W^{1,2}\left(0, T ; L^{2}(\Gamma)\right)$ we have that

$$
\int_{0}^{T-\delta}\left\|\tilde{w}_{\varepsilon}(t+\delta)-\widetilde{w}_{\varepsilon}(t)\right\|_{L^{2}(\Gamma)}^{2} d t \leq C|\delta|^{2}, \quad \forall \delta \in(0, T),
$$

(see [1]).

By surjectivity of the trace operator, this inequality remains true for $\widetilde{w}_{\varepsilon}$ in $H^{1}(\Omega)$ norm and we have that

$$
\begin{aligned}
& \int_{0}^{T-\delta}\left\|\tilde{w}_{\varepsilon}(t+\delta)-\tilde{w}_{\varepsilon}(t)\right\|_{L^{2}(\Omega)}^{2} d t \\
& \quad \leq C \int_{0}^{T-\delta}\left\|\tilde{w}_{\mathcal{\varepsilon}}(t+\delta)-\tilde{w}_{\varepsilon}(t)\right\|_{L^{2}(\Gamma)}^{2} d t \leq C|\delta|^{2}
\end{aligned}
$$

and so

$$
\widetilde{w}_{\varepsilon} \in W^{1,2}\left(0, T ; L^{2}(\Omega)\right) .
$$

We define

$$
\phi=w_{\varepsilon}-\tilde{w}_{\varepsilon}
$$

so that the problem (2.98)-(2.102) becomes

$$
\begin{gathered}
\frac{\partial \phi}{\partial t}-\Delta\left(\beta_{\varepsilon}\left(\theta_{\varepsilon}\right) \phi\right)+\frac{\partial}{\partial x_{3}}\left(K^{\prime}\left(\theta_{\varepsilon}\right) \phi\right)=f_{\phi}, \\
\phi=0 \quad \text { on } \Sigma_{u}, \quad \phi=0 \quad \text { on } \Sigma_{b}, \\
\left(K^{\prime}\left(\theta_{\varepsilon}\right) \phi i_{3}-\nabla\left(\beta_{\varepsilon}\left(\theta_{\varepsilon}\right) \phi\right)\right) \cdot v=\alpha \beta_{\varepsilon}\left(\theta_{\varepsilon}\right) \phi+f_{0 \phi} \quad \text { on } \Sigma_{\text {lat }}, \\
\phi(x, 0)=\phi_{0}(x) \quad \text { in } \Omega,
\end{gathered}
$$

where

$$
\begin{gathered}
f_{\phi}=f_{x_{3}}-\frac{\partial \tilde{w}_{\varepsilon}}{\partial t}+\Delta \beta_{\varepsilon}\left(\theta_{\varepsilon}\right)-\frac{\partial}{\partial x_{3}}\left(K^{\prime}\left(\theta_{\varepsilon}\right) \tilde{w}_{\varepsilon}\right), \\
f_{0 \phi}=-\left(K^{\prime}\left(\theta_{\varepsilon}\right) \tilde{w}_{\varepsilon} i_{3} \cdot \nu-\nabla\left(\beta_{\varepsilon}\left(\theta_{\varepsilon}\right) \tilde{w}_{\varepsilon}\right) \cdot \nu\right)+\alpha_{x_{3}} \beta_{\varepsilon}^{*}\left(\theta_{\varepsilon}\right)+\left(f_{0}\right)_{x_{3}} \quad \text { on } \Sigma_{\text {lat }}
\end{gathered}
$$

and $\phi_{0}(x)=w_{\varepsilon}(x, 0)-\widetilde{w}_{\varepsilon}(x, 0) \in L^{2}(\Omega)$.

We mention that under the specified assumptions

$$
f_{\phi} \in L^{2}\left(0, T ; L^{2}(\Omega)\right), \quad f_{0 \phi} \in L^{2}\left(0, T ; L^{2}\left(\Gamma_{\text {lat }}\right)\right) .
$$

We consider the space

$$
V_{0}=\left\{\psi \in H^{1}(\Omega) ; \psi=0 \text { on } \Gamma_{u}, \psi=0 \text { on } \Gamma_{b}\right\}
$$


with the usual norm and its dual denoted $V_{0}^{\prime}$ and we define the linear operator $A(t)$ : $V_{0} \rightarrow V_{0}^{\prime}$ by

$$
\begin{aligned}
\langle A(t) \phi, \psi\rangle= & \int_{\Omega}\left(\nabla\left(\beta_{\varepsilon}\left(\theta_{\varepsilon}(t)\right) \phi\right) \cdot \nabla \psi-K^{\prime}\left(\theta_{\varepsilon}(t)\right) \phi \frac{\partial \psi}{\partial x_{3}}\right) d x \\
& +\int_{\Gamma_{\text {lat }}} \alpha \beta_{\varepsilon}\left(\theta_{\varepsilon}(t)\right) \phi \psi d \sigma
\end{aligned}
$$

for any $\psi \in V_{0}$.

The operator $A(t)$ is bounded and coercive. Indeed, we have

$$
\begin{aligned}
\langle A(t) \phi, \phi\rangle= & \int_{\Omega}\left(\beta_{\varepsilon}\left(\theta_{\varepsilon}\right)|\nabla \phi|^{2}+\beta_{\varepsilon}^{\prime}\left(\theta_{\varepsilon}\right) \phi \nabla \theta_{\varepsilon} \cdot \nabla \phi-K^{\prime}\left(\theta_{\varepsilon}\right) \phi \frac{\partial \phi}{\partial x_{3}}\right) d x \\
& +\int_{\Gamma_{\text {lat }}} \alpha \beta_{\varepsilon}\left(\theta_{\varepsilon}\right) \phi^{2} d \sigma \geq \beta_{m}\|\phi\|_{V_{0}}^{2}+\alpha_{m} \beta_{m}\|\phi\|_{L^{2}\left(\Gamma_{\text {lat }}\right)}^{2} \\
& -\beta_{M}^{\prime}(\varepsilon)\left\|\phi \nabla \theta_{\varepsilon}\right\|\|\nabla \phi\|-M\|\phi\|\|\phi\|_{V_{0}} \\
\geq & \frac{\beta_{m}}{2}\|\phi\|_{V_{0}}^{2}+\alpha_{m} \beta_{m}\|\phi\|_{L^{2}\left(\Gamma_{\text {lat }}\right)}^{2}-\frac{M^{2}}{\beta_{m}}\|\phi\|^{2}-\frac{\left(\beta_{M}^{\prime}(\varepsilon)\right)^{2}}{\beta_{m}}\left\|\phi \nabla \theta_{\varepsilon}\right\|^{2} .
\end{aligned}
$$

Using (2.31) we calculate

$$
\begin{aligned}
\left\|\phi \nabla \theta_{\varepsilon}\right\|^{2} & =\int_{\Omega} \phi^{2}\left|\nabla \theta_{\varepsilon}\right|^{2} d x \leq\left(\int_{\Omega} \phi^{4} d x\right)^{1 / 2}\left(\int_{\Omega}\left|\nabla \theta_{\varepsilon}\right|^{4} d x\right)^{1 / 2} \\
& \leq C_{4}^{2}\|\phi(t)\|^{1 / 2}\|\phi(t)\|_{V_{0}}^{3 / 2}\left\|\nabla \theta_{\varepsilon}(t)\right\|^{1 / 2}\left\|\nabla \theta_{\varepsilon}(t)\right\|_{V_{0}}^{3 / 2} .
\end{aligned}
$$

But $\theta_{\varepsilon}$ satisfies (2.109) and so

$$
\left\|\phi \nabla \theta_{\varepsilon}\right\|^{2} \leq C(\varepsilon)\|\phi(t)\|^{1 / 2}\|\phi(t)\|_{V_{0}}^{3 / 2}
$$

Then we have

$$
\begin{aligned}
\frac{\left(\beta_{M}^{\prime}(\varepsilon)\right)^{2}}{\beta_{m}}\left\|\phi \nabla \theta_{\varepsilon}\right\|^{2} & \leq C(\varepsilon)\|\phi(t)\|^{1 / 2}\|\phi(t)\|_{V_{0}}^{3 / 2} \\
& \leq \frac{\beta_{m}}{4}\|\phi(t)\|_{V_{0}}^{2}+C(\varepsilon)\|\phi(t)\|^{2},
\end{aligned}
$$

where we applied relationship (2.73). Recalling (2.142) we obtain

$$
\langle A(t) \phi, \phi\rangle \geq \frac{\beta_{m}}{4}\|\phi\|_{V_{0}}^{2}+\alpha_{m} \beta_{m}\|\phi\|_{L^{2}\left(\Gamma_{\mathrm{lat}}\right)}^{2}-\left(\frac{M^{2}}{\beta_{m}}+C(\varepsilon)\right)\|\phi\|^{2} .
$$

By (2.141) we have

$$
\begin{aligned}
|A(t) \phi(\psi)| \leq & \beta_{M}(\varepsilon)\|\phi\|_{V_{0}}\|\psi\|_{V_{0}}+\beta_{M}(\varepsilon)\left\|\phi \nabla \theta_{\varepsilon}\right\|\|\psi\|_{V_{0}} \\
& +M\|\phi\|\|\psi\|_{V_{0}}+\alpha_{M} \beta_{M}(\varepsilon)\|\phi\|_{L^{2}\left(\Gamma_{\text {lat }}\right)}\|\psi\|_{L^{2}\left(\Gamma_{\text {lat }}\right)} .
\end{aligned}
$$


By (2.144) and Friedrichs' inequality we obtain

$$
\left\|\phi \nabla \theta_{\varepsilon}\right\| \leq C(\varepsilon)\|\phi(t)\|^{1 / 4}\|\phi(t)\|_{V_{0}}^{3 / 4} \leq C(\varepsilon)\|\phi(t)\|_{V_{0}}
$$

so that we finally can write (see (2.11) and (2.12)) that

$$
|A(t) \phi(\psi)| \leq\left(\beta_{M}(\varepsilon)+C(\varepsilon)\right)\|\phi\|_{V_{0}}\|\psi\|_{V_{0}} .
$$

In conclusion we deduce that

$$
\left.|A(t) \phi|\right|_{V_{0}^{\prime}} \leq C(\varepsilon)\|\phi\|_{V_{0}}
$$

so it is continuous. As previously, $C$ and $C(\varepsilon)$ denote various constants independent and dependent on $\varepsilon$, respectively. It follows that the operator $A(t)$ satisfies the hypotheses of Lions' theorem and since $f_{\phi} \in L^{2}\left(0, T ; V_{0}^{\prime}\right)$ and $\phi_{0} \in L^{2}(\Omega)$ we conclude that the system (2.133)-(2.136) has a unique solution

$$
\phi \in C\left([0, T] ; L^{2}(\Omega)\right) \cap L^{2}\left(0, T ; V_{0}\right), \quad \frac{d \phi}{d t} \in L^{2}\left(0, T ; V_{0}^{\prime}\right) .
$$

By (2.132) we obtain (2.107)-(2.108) as claimed.

2.3. Vertical monotonicity of the solution. To prove that there exists a free boundary $s=s\left(t, x_{1}, x_{2}\right)$ that determines a delimitation between the saturated domain denoted $Q_{+}=$ $\left\{(x, t) ; \theta(x, t)=\theta_{s}\right\}$ and the unsaturated one $Q_{-}=\left\{(x, t) ; \theta(x, t)<\theta_{s}\right\}$, with $Q_{+}$above $Q_{-}$, the idea is to prove that the function $\theta$ is monotonically decreasing with respect to $x_{3}$, that is, $w=\partial \theta / \partial x_{3} \leq 0$. Consequently, the equation $\theta(x, t)=\theta_{s}$ can be solved with respect to $x_{3}$ and yield a unique solution $x_{3}=s\left(t, x_{1}, x_{2}\right)$. Moreover, if $\theta \in C^{1}(Q)$, then the surface is smooth too.

We can now to pass to the proof of the vertical monotonicity of $\theta$ for the situation when $N=3, \alpha$ is constant and $f_{0}$ is time dependent only. First, we will prove the same thing for $\theta_{\varepsilon}$.

We use once again the approximate $\beta_{\varepsilon}^{*}$ given by (A.14).

For the case of interest in our problem, meaning the study of the top saturation occurrence $\left(\theta=\theta_{s}\right.$ on $\left.\Sigma_{u}\right)$ some supplementary conditions will be required and these include a monotonically vertical decreasing distribution of the initial data and source and some particular properties for the functions $u$ and $f_{0}$. So, first we will prove an intermediate result.

For each $\varepsilon>0$, let us introduce the functions $F_{\varepsilon}: \mathbb{R} \rightarrow \mathbb{R}$,

$$
F_{\varepsilon}(r)=K(r)-\alpha \beta_{\varepsilon}^{*}(r)
$$

and $F:\left(-\infty, \theta_{s}\right] \rightarrow \mathbb{R}$

$$
F(r)=K(r)-\alpha \stackrel{\circ}{\beta}(r)
$$

where $\stackrel{\circ}{\beta}^{*}(r)$ is the minimal section of $\beta^{*}$. This means that $F\left(\theta_{s}\right)=K_{s}^{*}$. 


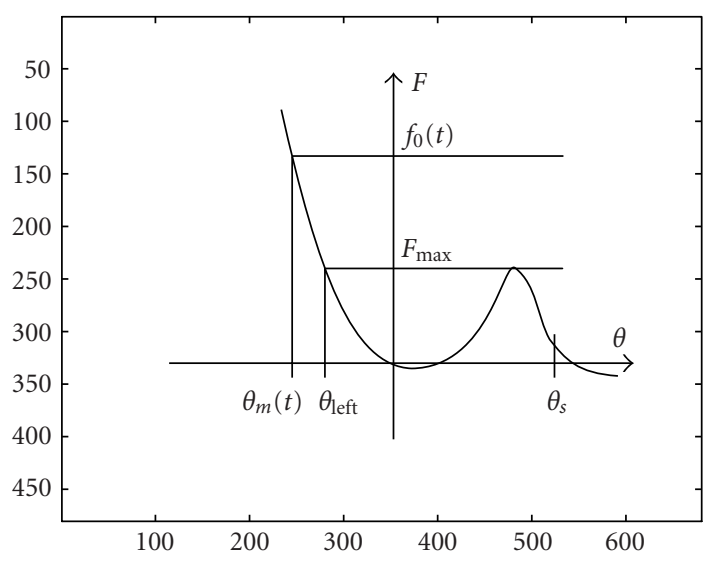

Figure 2.1. Determination of $\theta_{m}(t)$.

We notice that $F_{\varepsilon}$ is differentiable on $\mathbb{R}$ and $F$ is continuous (and differentiable on $\left.\left(-\infty, \theta_{s}\right)\right)$ and

$$
F(r)=F_{\varepsilon}(r), \quad \forall r \in\left(-\infty, \theta_{s}-\varepsilon\right)
$$

Denote by $F_{\min }=\min _{\theta \in\left[0, \theta_{s}\right]} F(\theta), F_{\max }=\max _{\theta \in\left[0, \theta_{s}\right]} F(\theta)$. Then

$$
F:\left(-\infty, \theta_{s}\right] \rightarrow\left[F_{\min },+\infty\right)
$$

Moreover, $F$ is strictly monotonically decreasing on $(-\infty, 0]$ because $F^{\prime}(r)=-\alpha \beta(r)<$ 0 for $r \leq 0$.

Lemma 2.8. Let $\alpha$ be a positive constant and $f_{0} \in C^{1}[0, T]$. Then, if $f_{0} \geq F_{\min }$, the equation

$$
F(r)=f_{0}(t)
$$

has at least one solution

$$
r(t)=F^{-1}\left(f_{0}(t)\right)
$$

for each $t$ fixed.

This follows by the continuity of the function $F:\left(-\infty, \theta_{s}\right] \rightarrow\left[F_{\min },+\infty\right)$.

Generally, $F^{-1}$ may be a multivalued function. Denote by $\theta_{\text {left }}$ the smallest solution to the equation $F(\theta)=F_{\max }$. We notice that if $f_{0}(t) \geq F_{\max }$, then the solution $F^{-1}\left(f_{0}(t)\right)$ is unique and it is smaller than $\theta_{\text {left }}$, see Figure 2.1. 
842 A free boundary problem

Lemma 2.9. Assume that the following conditions

$$
\begin{gathered}
f_{0} \in C^{1}[0, T], \quad f_{0}(t) \geq \sup _{\theta \in\left[0, \theta_{s}\right]} F(\theta), \\
f \in W^{1,2}\left(0, T ; L^{2}(\Omega)\right) \quad \text { a.e. on } Q, \\
\left(F^{-1}\left(f_{0}\right)\right)^{\prime}(t) \leq f \quad \text { a.e. on } Q, \\
u \in W^{1,2}\left(0, T ; L^{2}\left(\Gamma_{u}\right)\right) \cap L^{\infty}\left(0, T ; H^{1}\left(\Gamma_{u}\right)\right) \cap L^{\infty}\left(\Sigma_{u}\right), \\
K_{s} \leq-u \quad \text { a.e. on } \Sigma_{u}, \\
\theta_{0} \in H^{2}(\Omega), \quad 0 \leq \theta_{0} \leq \theta_{s}
\end{gathered}
$$

hold true. Then, there exists $\theta_{m}(t)$ independent of $\varepsilon$, such that the approximating solution $\theta_{\varepsilon}$ satisfies

$$
\begin{gathered}
\theta_{m}(t) \leq \theta_{\varepsilon}(x, t) \quad \text { a.e. on } \Omega, \text { for any } t \in[0, T], \varepsilon>0, \\
\frac{\partial \theta_{\varepsilon}}{\partial x_{3}}(x, t) \leq 0 \quad \text { a.e. on } \Sigma_{b}, \\
\frac{\partial \theta_{\varepsilon}}{\partial x_{3}}(x, t) \leq 0 \quad \text { a.e. on } \Sigma_{u} .
\end{gathered}
$$

Proof. By Theorem 1.1 the approximating solution $\theta_{\varepsilon}$ exists and satisfies the boundary condition (1.24) which particularly written on the part $\Sigma_{b}$ becomes

$$
\frac{\partial \theta_{\varepsilon}}{\partial x_{3}}=\frac{K\left(\theta_{\varepsilon}\right)-\alpha \beta_{\varepsilon}^{*}\left(\theta_{\varepsilon}\right)-f_{0}}{\beta_{\varepsilon}\left(\theta_{\varepsilon}\right)} \quad \text { a.e. on } \Sigma_{b} .
$$

Also

$$
\frac{\partial \theta_{\varepsilon}}{\partial x_{3}}=\frac{K\left(\theta_{\varepsilon}\right)+u}{\beta_{\varepsilon}\left(\theta_{\varepsilon}\right)} \quad \text { a.e. on } \Sigma_{u}
$$

Condition (2.158) implies that

$$
f_{0}(t) \geq F_{\max } \geq K\left(\theta_{\varepsilon}\right)-\alpha \stackrel{\circ *}{\beta}\left(\theta_{\varepsilon}\right), \quad \forall \theta_{\varepsilon} \in\left[0, \theta_{s}\right],
$$

in particular

$$
f_{0}(t) \geq K_{s}-\alpha K_{s}^{*}
$$

Moreover, since

$$
F\left(\theta_{\varepsilon}\right)=K\left(\theta_{\varepsilon}\right)-\alpha \beta^{*}\left(\theta_{\varepsilon}\right) \geq K\left(\theta_{\varepsilon}\right)-\alpha \beta_{\varepsilon}^{*}\left(\theta_{\varepsilon}\right), \quad \forall \theta_{\varepsilon} \in\left[0, \theta_{s}\right),
$$

(see $\left(\right.$ A.22)) and $F\left(\theta_{s}\right)=F_{\varepsilon}\left(\theta_{s}\right)$ we have that

$$
f_{0}(t) \geq \sup _{\theta_{\varepsilon} \in\left[0, \theta_{s}\right]} F\left(\theta_{\varepsilon}\right) \geq K\left(\theta_{\varepsilon}\right)-\alpha \beta_{\varepsilon}^{*}\left(\theta_{\varepsilon}\right) \quad \text { on } \Sigma_{b}, \forall \theta_{\varepsilon} \in\left[0, \theta_{s}\right] .
$$


If $\theta_{\varepsilon}>\theta_{s}$ then $\beta_{\varepsilon}^{*}\left(\theta_{\varepsilon}\right)>K_{s}^{*}$ and by $(2.170)$ we get

$$
f_{0}(t) \geq K_{s}-\alpha K_{s}^{*}>K\left(\theta_{\varepsilon}\right)-\alpha \beta_{\varepsilon}^{*}\left(\theta_{\varepsilon}\right), \quad \forall \theta_{\varepsilon}>\theta_{s} .
$$

Now, for each $t$ fixed the horizontal $y=f_{0}(t) \geq F_{\max }$ intersects the graphic of the function $y=F(\theta)$ yet at one point situated on the left decreasing branch of $F$ (see Figure 2.1).

Hence, for each $t$ fixed, we define

$$
\theta_{m}(t)=\min \left\{r_{j}(t), F\left(r_{j}(t)\right)=f_{0}(t)\right\}
$$

and $\theta_{m}$ follows to be independent on $\varepsilon$.

By the decreasing monotonicity of $F$ we obtain that if $f_{0}(t)=F\left(\theta_{m}\right) \geq F_{\max }$ then $\theta_{m}(t) \leq$ $\theta_{\text {left }}$, where $\theta_{\text {left }}$ is the smallest solution to $F_{\max }=f_{0}(t)$.

Moreover, it follows that $t \rightarrow \theta_{m}(t)$ is differentiable,

$$
\theta_{m}^{\prime}(t)=\frac{1}{F^{\prime}\left(F^{-1}\left(f_{0}(t)\right)\right)} .
$$

In particular, if $F_{\max } \geq 0$, then $\theta_{m}(t) \leq 0$, whence we obtain

$$
f_{0}(t) \geq F_{\max } \geq F\left(\theta_{\varepsilon}\right)=F_{\varepsilon}\left(\theta_{\varepsilon}\right) \text { for } \theta_{\varepsilon} \in\left[\theta_{m}(t), 0\right) .
$$

Finally we get from (2.172), (2.173), and (2.176) that assumption (2.158) turns out in

$$
f_{0}(t) \geq F\left(\theta_{\varepsilon}\right) \text { for } \theta_{m}(t) \leq \theta_{\varepsilon} \text { on } \Sigma_{b}
$$

and for each $t$ we have

$$
f_{0}(t)=F\left(\theta_{m}(t)\right)=\sup _{\theta \geq \theta_{m}(t)} F(\theta)
$$

Further, using (2.160) we can write

$$
\theta_{m}^{\prime}(t) \leq f \quad \text { on } Q
$$

Then, by (2.162) we have

$$
K\left(\theta_{m}(t)\right) \leq K_{s} \leq-u \quad \text { on } \Sigma_{u}
$$

because $K$ is a monotonically increasing function for $\theta \leq \theta_{s}$ and $\theta_{m}(t)<\theta_{s}$.

In conclusion by (2.158), (2.163), (2.179), (2.180) and Lemma 1.3 it follows that

$$
\theta_{m}(t) \leq \theta_{\varepsilon}(x, t) \quad \text { a.e. on } \Omega, \forall t \in[0, T] .
$$

By (2.167) and (2.164) we successively get

$$
\begin{gathered}
\frac{\partial \theta_{\varepsilon}}{\partial x_{3}}=\frac{K\left(\theta_{\varepsilon}\right)-\alpha \beta_{\varepsilon}^{*}\left(\theta_{\varepsilon}\right)-f_{0}(t)}{\beta_{\varepsilon}\left(\theta_{\varepsilon}\right)} \leq \frac{\sup _{\theta_{\varepsilon} \geq \theta_{m}(t)} F\left(\theta_{\varepsilon}\right)-f_{0}(t)}{\beta_{\varepsilon}\left(\theta_{\varepsilon}\right)} \text { a.e. on } \Sigma_{b}, \\
\frac{\partial \theta_{\varepsilon}}{\partial x_{3}} \leq \frac{F\left(\theta_{m}(t)\right)-f_{0}(t)}{\beta_{\varepsilon}\left(\theta_{\varepsilon}\right)} \text { a.e. on } \Sigma_{b},
\end{gathered}
$$


844 A free boundary problem

that is, (2.165). Analogously we obtain

$$
\frac{\partial \theta_{\varepsilon}}{\partial x_{3}}=\frac{K\left(\theta_{\varepsilon}\right)+u}{\beta_{\varepsilon}\left(\theta_{\varepsilon}\right)} \leq \frac{K_{s}+u}{\beta_{\varepsilon}\left(\theta_{\varepsilon}\right)} \leq 0 \quad \text { a.e. on } \Sigma_{u}
$$

as claimed.

Theorem 2.10. Assume the hypotheses of Theorem 2.7 and Lemma 2.9, that is,

$$
\begin{gathered}
\theta_{0} \in H^{2}(\Omega), \\
0 \leq \theta_{0} \leq \theta_{s} \text { a.e. on } \Omega, \\
u \in W^{1,2}\left(0, T ; L^{2}\left(\Gamma_{u}\right)\right) \cap L^{\infty}\left(0, T ; H^{1}\left(\Gamma_{u}\right)\right) \cap L^{\infty}\left(\Sigma_{u}\right), \\
K_{s} \leq-u \quad \text { a.e. on } \Sigma_{u}, \\
f_{0} \in C^{1}[0, T], \\
f_{0}(t) \geq \sup _{\theta \in\left[0, \theta_{s}\right]}(K(\theta)-\alpha \stackrel{\circ}{\beta}(\theta)), \\
f \in W^{1,2}\left(0, T ; L^{2}(\Omega)\right) \quad \text { a.e. on } Q, \\
\left(F^{-1}\left(f_{0}\right)\right)^{\prime}(t) \leq f,
\end{gathered}
$$

and $\alpha>0$,

$$
\begin{array}{cc}
\frac{\partial \theta_{0}}{\partial x_{3}}(z, 0) \leq 0 & \text { a.e. in } \Omega \\
f_{x_{3}}(x, t) & \leq 0 \quad \text { a.e. in } Q .
\end{array}
$$

Then $w=\partial \theta / \partial z$ is negative a.e. on $\Omega \times(0, T)$ and $x_{3} \rightarrow \theta\left(x_{1}, x_{2}, x_{3}, t\right)$ is monotonically decreasing on $[0, T]$ for each $t \in[0, T]$.

Proof. We recall system (2.98)-(2.102) for the derivative $w_{\varepsilon}=\partial \theta_{\varepsilon} / \partial x_{3}$ in the following form

$$
\begin{gathered}
\frac{\partial w_{\varepsilon}}{\partial t}-\Delta\left(\beta_{\varepsilon}\left(\theta_{\varepsilon}\right) w_{\varepsilon}\right)+\frac{\partial}{\partial x_{3}}\left(K^{\prime}\left(\theta_{\varepsilon}\right) w_{\varepsilon}\right)=f_{x_{3}}, \\
w_{\varepsilon}(x, 0)=w_{0}(x) \text { in } \Omega, \\
K\left(\theta_{\varepsilon}\right)-\beta_{\varepsilon}\left(\theta_{\varepsilon}\right) w_{\varepsilon}=-u \quad \text { on } \Sigma_{u}, \\
K\left(\theta_{\varepsilon}\right)-\beta_{\varepsilon}\left(\theta_{\varepsilon}\right) w_{\varepsilon}=\alpha \beta_{\varepsilon}^{*}\left(\theta_{\varepsilon}\right)+f_{0} \quad \text { on } \Sigma_{b}, \\
\left(K^{\prime}\left(\theta_{\varepsilon}\right) w_{\varepsilon} i_{3}-\nabla\left(\beta_{\varepsilon}\left(\theta_{\varepsilon}\right) w_{\varepsilon}\right)\right) \cdot v=\alpha \beta_{\varepsilon}\left(\theta_{\varepsilon}\right) w_{\varepsilon} \quad \text { on } \Sigma_{\text {lat }} .
\end{gathered}
$$

Under the hypotheses (2.184) the approximating solution $\theta_{\varepsilon}$ has, by Theorem 2.5 , the properties

$$
\begin{gathered}
\theta_{\varepsilon} \in W^{1, \infty}\left(0, T ; L^{2}(\Omega)\right) \cap W^{1,2}(0, T ; V) \cap L^{\infty}\left(0, T ; H^{2}(\Omega)\right), \\
\beta_{\varepsilon}^{*}\left(\theta_{\varepsilon}\right) \in W^{1, \infty}\left(0, T ; L^{2}(\Omega)\right) \cap W^{1,2}(0, T ; V) \cap L^{\infty}\left(0, T ; H^{2}(\Omega)\right) .
\end{gathered}
$$


Moreover, due to Lemma 2.9, we have that there exists $\theta_{m}(t)$ such that $\theta_{m}(t) \leq \theta_{\varepsilon}$,

$$
\frac{\partial \theta_{\varepsilon}}{\partial x_{3}} \leq 0 \quad \text { a.e. on } \Sigma_{b}, \quad \frac{\partial \theta_{\varepsilon}}{\partial x_{3}} \leq 0 \quad \text { a.e. on } \Sigma_{u}
$$

implying that $w_{\varepsilon}^{+}=0$ a.e. on $\Sigma_{b}$ and on $\Sigma_{u}$.

With these considerations, we can multiply (2.186) by $w_{\varepsilon}^{+}$and integrate it over $\Omega \times$ $(0, t)$. We have

$$
\begin{aligned}
\int_{0}^{t} \int_{\Omega} \frac{\partial w_{\varepsilon}}{\partial t} w_{\varepsilon}^{+} d x d \tau+\int_{0}^{t} \int_{\Omega} \nabla\left(\beta_{\varepsilon}\left(\theta_{\varepsilon}\right) w_{\varepsilon}\right) \cdot \nabla w_{\varepsilon}^{+} d x d \tau \\
=\int_{0}^{t} \int_{\Omega} K^{\prime}\left(\theta_{\varepsilon}\right) w_{\varepsilon} \frac{\partial w_{\varepsilon}^{+}}{\partial x_{3}} d x d t-\int_{0}^{t} \int_{\Gamma_{b}}\left(\alpha \beta_{\varepsilon}^{*}\left(\theta_{\varepsilon}\right)+f_{0}\right) w_{\varepsilon}^{+} d \sigma d \tau \\
\quad+\int_{0}^{t} \int_{\Gamma_{u}} u w_{\varepsilon}^{+} d \sigma d \tau-\int_{0}^{t} \int_{\Gamma_{\mathrm{lat}}} \alpha \beta_{\varepsilon}\left(\theta_{\varepsilon}\right) w_{\varepsilon} w_{\varepsilon}^{+} d \sigma d \tau+\int_{0}^{t} \int_{\Omega} f_{x_{3}} w_{\varepsilon}^{+} d x_{3} d \tau .
\end{aligned}
$$

We have

$$
\begin{aligned}
\frac{1}{2} \int_{0}^{t} \int_{\Omega} & \frac{\partial\left(w_{\varepsilon}^{+}\right)^{2}}{\partial t} d x d \tau+\int_{0}^{t} \int_{\Omega} \beta_{\varepsilon}\left(\theta_{\varepsilon}\right)\left|\nabla w_{\varepsilon}^{+}\right|^{2} d x d \tau \\
& +\int_{0}^{t} \int_{\Gamma_{\mathrm{lat}}} \alpha \beta_{\varepsilon}\left(\theta_{\varepsilon}\right)\left(w_{\varepsilon}^{+}\right)^{2} d \sigma d \tau+\int_{0}^{t} \int_{\Omega} \beta_{\varepsilon}^{\prime}\left(\theta_{\varepsilon}\right) w_{\varepsilon} \nabla \theta_{\varepsilon} \cdot \nabla w_{\varepsilon}^{+} d x d \tau \\
\leq & M \int_{0}^{t}\left\|w_{\varepsilon}^{+}(\tau)\right\|\left\|\frac{\partial w_{\varepsilon}^{+}}{\partial x_{3}}(\tau)\right\| d \tau .
\end{aligned}
$$

The rest of the terms on the right-hand side vanishes, due to the considered hypotheses. Then we have

$$
\begin{aligned}
& \int_{0}^{t} \int_{\Omega} \beta_{\varepsilon}\left(\theta_{\varepsilon}\right) w_{\varepsilon} \nabla \theta_{\varepsilon} \cdot \nabla w_{\varepsilon}^{+} d x d \tau \\
& \quad \leq \int_{0}^{t}\left\|\beta_{\varepsilon}^{\prime}\left(\theta_{\varepsilon}(\tau)\right) w_{\varepsilon}^{+}(\tau) \nabla \theta_{\varepsilon}(\tau)\right\|\left\|\nabla w_{\varepsilon}^{+}(\tau)\right\| d \tau \\
& \quad \leq \frac{1}{2}\left(\int_{0}^{t} \frac{2 \beta_{M}^{\prime}(\varepsilon)}{\beta_{m}}\left\|w_{\varepsilon}^{+}(\tau) \nabla \theta_{\varepsilon}(\tau)\right\|^{2} d \tau+\frac{\beta_{m}}{2} \int_{0}^{t}\left\|\nabla w_{\varepsilon}^{+}(\tau)\right\|^{2} d \tau\right)
\end{aligned}
$$

and noticing that by hypothesis $w_{\varepsilon}^{+}(0)=0$, we deduce that

$$
\begin{gathered}
\frac{1}{2}\left\|w_{\varepsilon}^{+}(t)\right\|^{2}+\frac{\beta_{m}}{2} \int_{0}^{t}\left\|\left(\nabla w_{\varepsilon}(\tau)\right)^{+}\right\|^{2} d \tau+\int_{0}^{t} \int_{\Gamma_{\mathrm{lat}}} \alpha \beta_{\varepsilon}\left(\theta_{\varepsilon}\right)\left(w_{\varepsilon}^{+}\right)^{2} d \sigma d \tau \\
\leq \frac{M^{2}}{\beta_{m}} \int_{0}^{t}\left\|w_{\varepsilon}^{+}(\tau)\right\|^{2} d \tau+\frac{\beta_{M}^{\prime}(\varepsilon)}{\beta_{m}} \int_{0}^{t}\left\|w_{\varepsilon}^{+}(\tau) \nabla \theta_{\varepsilon}(\tau)\right\|^{2} d \tau .
\end{gathered}
$$


Taking into account that $\partial \theta_{\varepsilon} / \partial x_{i} \in L^{\infty}(0, T ; V)$ we have

$$
\begin{aligned}
\int_{\Omega}( & \left.w_{\varepsilon}^{+}(\tau)\right)^{2}\left|\nabla \theta_{\varepsilon}(\tau)\right|^{2} d x \\
& \leq\left(\int_{\Omega}\left(w_{\varepsilon}^{+}\right)^{4}(\tau) d x\right)^{1 / 2}\left(\int_{\Omega}\left|\nabla \theta_{\varepsilon}(\tau)\right|^{4} d x\right)^{1 / 2} \\
& \leq C_{4}^{2}\left\|\left(w_{\varepsilon}^{+}\right)(\tau)\right\|^{1 / 2}\left\|\left(w_{\varepsilon}^{+}\right)(\tau)\right\|_{H^{1}(\Omega)}^{3 / 2}\left\|\nabla \theta_{\varepsilon}(\tau)\right\|^{1 / 2}\left\|\nabla \theta_{\varepsilon}(\tau)\right\|_{H^{1}(\Omega)}^{3 / 2} \\
& \leq \frac{\beta_{m}}{4}\left\|\left(\nabla w_{\varepsilon}(\tau)\right)^{+}\right\|^{2}+C(\varepsilon)\left\|w_{\varepsilon}^{+}(\tau)\right\|^{2},
\end{aligned}
$$

(see (2.31) and (2.145)). From (2.192) we get finally that

$$
\left\|w_{\varepsilon}^{+}(t)\right\|^{2} \leq C(\varepsilon) \int_{0}^{t}\left\|w_{\varepsilon}^{+}(\tau)\right\|^{2} d \tau
$$

which implies, by Gronwall's lemma that $w_{\varepsilon}^{+}(x, t)=0$ a.e. on $\Omega$, for each $t \in[0, T]$.

Consequently, the function $x_{3} \rightarrow \theta_{\varepsilon}(x, t)$ is decreasing, meaning that if $x_{3}, x_{3}^{\prime} \in[0, L]$ with $x_{3}<x_{3}^{\prime}$ it follows that

$$
\theta_{\varepsilon}\left(x_{1}, x_{2}, x_{3}, t\right) \leq \theta_{\varepsilon}\left(x_{1}, x_{2}, x_{3}^{\prime}, t\right)
$$

This inequality is preserved by passing to limit as $\varepsilon \rightarrow 0$ so, we find that $x_{3} \rightarrow \theta(x, t)$ is decreasing a.e. on $(0, L)$.

The previous result enables us to state.

COROLlaRY 2.11. There is a graph $x_{3}=s\left(x_{1}, x_{2}, t\right)$ that separates the saturated region $Q_{+}$by the unsaturated region $Q_{-}$.

Proof. By Theorem 2.7 we may conclude that either the flow remains all the time unsaturated if the saturation did not occur first at the surface or there exists only one saturated subdomain and only one unsaturated separated by the free boundary s. Indeed, if $\theta\left(x_{1}, x_{2}, 0, t\right)<\theta_{s}$, for all $t$ and all $\left(x_{1}, x_{2}\right) \in D$, then $\theta\left(x_{1}, x_{2}, x_{3}, t\right)<\theta_{s}$ a.e. $x \in \Omega$.

If there exists $t_{s}>0$ and $\left(x_{1}^{s}, x_{2}^{s}\right)$ such that $\theta\left(x_{1}^{s}, x_{2}^{s}, 0, t_{s}\right)=\theta_{s}$, then by the monotonicity of $\theta$ we have that $\theta\left(x_{1}^{s}, x_{2}^{s}, x_{3}, t_{s}\right) \leq \theta_{s}, \forall x_{3} \geq 0$. The equality may take place for $x_{3} \in\left[0, x_{3}^{s}\right]$ while for $x_{3} \in\left(x_{3}^{s}, L\right]$ we have the strict inequality.

Then, the proof of the corollary is immediate by defining

$$
s\left(x_{1}, x_{2}, t\right)=\sup \left\{x_{3} ; \theta\left(x_{1}, x_{2}, x_{3}, t\right)=\theta_{s}\right\}
$$

or $s\left(x_{1}, x_{2}, t\right)=\inf \left\{x_{3} ; \theta\left(x_{1}, x_{2}, x_{3}, t\right)<\theta_{s}\right\}$.

We mention also that in the case when $\theta$ is smooth then the free surface is smooth too. 


\section{Weak solution}

We recall now the infiltration boundary value problem written in pressure form (see Appendix A.1)

$$
\begin{gathered}
C(h) \frac{\partial h}{\partial t}-\nabla \cdot(k(h) \nabla h)+\frac{\partial k(h)}{\partial x_{3}}=f \quad \text { in } Q, \\
h(x, 0)=h_{0}(x) \quad \text { in } \Omega, \\
\left(k(h) i_{3}-k(h) \nabla h\right) \cdot v=u \quad \text { on } \Sigma_{u}, \\
\left(k(h) i_{3}-k(h) \nabla h\right) \cdot v=\alpha K^{*}(h)+f_{0} \quad \text { on } \Sigma_{\alpha}
\end{gathered}
$$

and remind the definition of the weak solution to (3.1)-(3.4) and the result that states the equivalence between this model and that one describing the occurrence of the saturatedunsaturated domains $Q_{+}$and $Q_{-}$(see [9]).

Let $V$ be the space $H^{1}(\Omega)$ endowed with the usual Hilbertian norm.

Definition 3.1. The function $h \in L^{2}\left(0, T ; L^{2}(\Omega)\right)$ is said to be a weak solution to problem (3.1)-(3.4) if $K^{*}(h) \in L^{2}(0, T ; V)$ and

$$
\begin{aligned}
\int_{Q}(- & \left.C^{*}(h) \phi_{t}(x, t)+\nabla K^{*}(h) \cdot \nabla \phi(x, t)-k(h) \frac{\partial \phi}{\partial x_{3}}(x, t)\right) d x d t \\
= & \int_{\Omega} \phi(x, 0) C^{*}\left(h_{0}(x)\right) d x-\int_{\Sigma_{\alpha}}\left(\alpha(x) K^{*}(h)+f_{0}(x, t)\right) \phi(x, t) d \sigma d t \\
& -\int_{\Sigma_{u}} u(x, t) \phi(x, t) d \sigma d t+\int_{Q} f(x, t) \phi(x, t) d x d t
\end{aligned}
$$

for all $\phi \in L^{2}\left(0, T ; H^{1}(\Omega)\right)$ with $\phi_{t} \in L^{2}\left(0, T ; L^{2}(\Omega)\right)$ and $\phi(x, T) \equiv 0$.

Proposition 3.2 (see [9]). Assume

$$
\begin{gathered}
f \in L^{2}\left(0, T ; V^{\prime}\right), \quad u \in L^{2}\left(0, T ; L^{2}\left(\Gamma_{u}\right)\right), \quad f_{0} \in L^{2}\left(0, T ; L^{2}\left(\Gamma_{\alpha}\right)\right), \\
\theta_{0} \in L^{2}(\Omega), \quad \theta_{0} \leq \theta_{s} \text { a.e. on } \Omega .
\end{gathered}
$$

Then problem (3.1)-(3.4) has a weak solution $h \in L^{2}(0, T ; V)$.

Remark 3.3. By the strictly increasing monotonicity of the function $h \rightarrow C^{*}(h)$ on $h<0$ we obtain, under the appropriate conditions of Lemma 1.3, that if $\theta \geq \theta_{r}$, then $h \geq h_{r}$, so $h$ belongs also to the physical domain.

In terms of pressure we set $Q_{-}=\{(x, t) ; h(x, t)<0\}, Q_{+}=\{(x, t) ; h(x, t)>0\}$, and $Q_{0}=$ $\{(x, t) ; h(x, t)=0\}$ for the free surface. 
Proposition 3.4. Assume that $Q_{-}, Q_{+}$are open and $Q_{0}$ is a smooth surface. Then, the weak and smooth solution to (3.1)-(3.4) is a solution to the below model describing the water infiltration into an unsaturated-saturated soil

$$
\begin{gathered}
C(h) \frac{\partial h}{\partial t}-\Delta K^{*}(h)+\frac{\partial k(h)}{\partial x_{3}}=f \quad \text { in } Q_{-}, \\
-K_{s} \Delta h=f \quad \text { in } Q_{+}, \\
h(x, 0)=h_{0}(x) \quad \text { in } \Omega, \\
q^{+}(x, t)=q^{-}(x, t) \quad \text { on } Q_{0}, \quad h^{+}(x, t)=h^{-}(x, t)=0 \quad \text { on } Q_{0}, \\
q \cdot v=u(x, t) \quad \text { on } \Sigma_{u}, \quad q \cdot v=\alpha(x) K^{*}(h)+f_{0}(x, t) \quad \text { on } \Sigma_{\alpha} .
\end{gathered}
$$

Here, $q^{ \pm}, h^{ \pm}$are the limit values of $q$ and $h$ from the sides $Q^{ \pm}$. Now we can prove

Theorem 3.5. The weak and continuous solution to (3.1)-(3.4) is unique.

Proof. The uniqueness in the 1D unsaturated domain was proved in [9]. For the 3D case we consider two solutions $h$ and $\bar{h}$, satisfying (3.5) and let consider the relation satisfied by their difference. For continuous solutions $h$, the sets $Q_{+}$and $Q_{-}$are open and taking $\psi$ with compact support in the interior of $Q_{+}$we get

$$
\int_{Q_{+}} \nabla K_{s}(h-\bar{h}) \cdot \nabla \psi d x d t=-\int_{\Sigma_{\alpha}} \alpha K_{s}(h-\bar{h}) \psi d \sigma d t
$$

where $K^{*}(h)=K_{s}^{*}+K_{s} h$. Setting now $\psi=h-\bar{h}$ we obtain

$$
0 \leq \int_{Q_{+}}|\nabla(h-\bar{h})|^{2} d x d t=-\int_{\Sigma_{\alpha}} \alpha(h-\bar{h})^{2} d \sigma d t
$$

implying that $h=\bar{h}$, that is, the pressure uniqueness in the saturated domain.

\section{Conclusions}

The present paper, as well as paper [9] have been intended to develop a functional approach for the problem of simultaneous saturated-unsaturated flow into a porous medium. The modeling of the process was done in order to meet the particularities of the most used hydraulic models in soil sciences, for example that of Broadbridge-White and van Genuchten, with a blowing-up diffusivity at saturation occurrence. The problem of mathematical modeling of the flow around the saturation point was solved by the introduction of the multivalued function (1.5).

Under assumptions on the initial and boundary data appropriate to those existent in a real concrete problem the existence of the simultaneous saturated-unsaturated flow was proved. Further, for realistic conditions, as a positively distributed initial moisture, bounded sources and rain rates, the boundedness of the solution and its placement in a physical accepted domain for moistures were proved. 
Also, by the comparison of the solution with known regular time functions, a forecast of the flow behavior can be done, for a given structure of the initial and boundary data.

During the phenomenon evolution the formation of saturated zones may be observed anywhere in the flow domain. But due to particular flow conditions, situations in which two separated domains are formed, one saturated and the other unsaturated may occur. Experimentally it was put in evidence that, at least in the 1D case, the apparition of the saturation at a certain first place, is related to the ratio between the rain rate and the conductivity at saturation. If this ratio is less than 1 then first saturates the basement and the water front advances to the top and if it is greater that 1 then saturation is formed beginning from the soil surface (see [7]).

Besides the condition concerning this ratio, in the paper sufficient conditions under which the latter situation may appear are given. Consequently, a definition of the free boundary was introduced. However its regularity remains an open problem.

Finally a discussion on the solution in pressure form was made. In mechanics one deals with the systems written in the forms (3.1)-(3.4), and (3.7)-(3.11) which corresponds to the already separated flows. In order to give a mathematical meaning to the first, the definition of the weak solution was introduced and a result of existence for this solution was proved. Further a rigorous link between these two models has been done in Proposition 3.4 proved for smooth solutions.

\section{Appendix}

\section{A.1. Review of the model.}

Description of the hydraulic model. We consider that infiltration is described by the boundary value problem written in the pressure form (3.1)-(3.4), in which the hydraulic model (represented by the hydraulic conductivity $k$ and constitutive law $\theta$ (moisture)) is defined as follows in terms of the pressure $h$ :

$\left(\mathrm{a}_{1}\right) k:\left[h_{r}, 0\right] \rightarrow\left[K_{r}, K_{s}\right] ; k$ is positive, monotonically increasing, twice differentiable, convex on $\left[K_{r}, K_{s}\right]$ and $k^{\prime}\left(h_{r}\right)=0$;

$\left(\mathrm{a}_{2}\right) \theta:\left[h_{r}, 0\right] \rightarrow\left[\theta_{r}, \theta_{s}\right] ; \theta$ is positive, monotonically increasing, twice differentiable and concave.

The value $\theta_{s}$ is the value of moisture at saturation and $\theta_{r}=\theta\left(h_{r}\right),\left(h_{r}<0\right)$ is the residual value of moisture, meaning the moisture resident in a dried soil. Practically, under this value there is no flow. The values $K_{s}$ and $K_{r}$ are the values of conductivity at saturation and respectively for a dried soil. The positive values $\theta_{r}, \theta_{s}, K_{r}, K_{s}$ are soil characteristics and they are known. The condition $k^{\prime}\left(h_{r}\right)=0$ was put in evidence in experiments (see [6]).

The derivative of $\theta$ with respect to $h$ is called water capacity, $C$.

$\left(\mathrm{a}_{3}\right) C:\left[h_{r}, 0\right] \rightarrow\left[0, C_{r}\right]$ is a positive, bounded, continuous, monotonically decreasing function on $\left[h_{r}, 0\right]$, and $C^{\prime}\left(h_{r}\right)=0$.

This means that the flow is defined up to the maximum capacity of the soil.

Moreover we assume

$\left(\mathrm{i}_{\mathrm{K}}\right)$ there exists $M>0$ such that $k^{\prime}(h) \leq M C(h)$.

At saturation we have

$\left(\mathrm{a}_{4}\right) \theta(h)=\theta_{s}, k(h)=K_{s}$ and $C(h)=0$, for $h \geq 0$. 
The diffusive model. We define the primitives of $C$ and $K$ by $C^{*}:\left[h_{r}, \infty\right) \rightarrow\left[\theta_{r}, \theta_{s}\right]$,

$$
\begin{aligned}
C^{*}(h) & = \begin{cases}\theta_{r}+\int_{h_{r}}^{h} C(\zeta) d \zeta, & h<0 \\
\theta_{s}, & h \geq 0,\end{cases} \\
K^{*}(h) & = \begin{cases}\int_{h_{r}}^{h} k(\zeta) d \zeta, & h<0 \\
K_{s}^{*}+K_{s} h, & h \geq 0, K_{s}^{*}=K^{*}(0)\end{cases}
\end{aligned}
$$

and notice that $\theta=C^{*}(h)$.

Both functions are continuous and monotonically increasing $\left(C^{*}\right.$ is strictly monotonically increasing on $h<0$ ) and with these notations Richards' equation (3.1) becomes

$$
\frac{\partial C^{*}(h)}{\partial t}-\Delta K^{*}(h)+\frac{\partial k(h)}{\partial x_{3}}=f \quad \text { in } Q .
$$

Being more convenient to work with the variable $\theta$, we introduce from (A.1) the inverse of $C^{*}$

$$
h= \begin{cases}\left(C^{*}\right)^{-1}(\theta), & \theta \in\left[\theta_{r}, \theta_{s}\right) \\ {[0,+\infty),} & \theta=\theta_{s}\end{cases}
$$

and replace all over in (A.3), (3.2)-(3.4) $h$ by (A.4). So we get the multivalued function

$$
\beta^{*}(\theta)= \begin{cases}K^{*}\left(\left(C^{*}\right)^{-1}(\theta)\right), & \theta \in\left[\theta_{r}, \theta_{s}\right), \\ {\left[K_{s}^{*},+\infty\right),} & \theta=\theta_{s}\end{cases}
$$

where $K_{s}^{*}=\lim _{\theta>\theta_{s}} \beta^{*}(\theta)$ and the conductivity expressed as function of $\theta$

$$
K(\theta)=k\left(\left(C^{*}\right)^{-1}(\theta)\right), \quad \theta \in\left[\theta_{r}, \theta_{s}\right] .
$$

For $\theta \in\left(\theta_{r}, \theta_{s}\right)$ we can calculate the derivative of $\beta^{*}(\theta)$, denoted by $\beta(\theta)$ which follows to be given by

$$
\beta(\theta)=\frac{k\left(\left(C^{*}\right)^{-1}(\theta)\right)}{C\left(\left(C^{*}\right)^{-1}(\theta)\right)}
$$

By the hypotheses made on the functions $C$ and $k$ it follows that $\beta$ is monotonically increasing and convex, so

$$
\begin{gathered}
\beta(\theta) \geq \beta\left(\theta_{r}\right):=\rho>0, \\
\beta^{\prime}(\theta)>0, \quad \beta^{\prime \prime}(\theta)>0, \quad \text { for } \theta \in\left[\theta_{r}, \theta_{s}\right]
\end{gathered}
$$

and $\beta^{\prime}\left(\theta_{r}\right)=0, K^{\prime}\left(\theta_{r}\right)=0$. 
By the assumptions made up to now we deduce that the functions $\beta^{*}$ and $K$ satisfy

(i) $\left(\beta^{*}(\theta)-\beta^{*}(\theta)\right)(\theta-\bar{\theta}) \geq \rho(\theta-\bar{\theta})^{2}, \forall \theta, \bar{\theta} \in\left(-\infty, \theta_{s}\right]$.

(ii) $\lim _{\theta \rightarrow-\infty} \beta^{*}(\theta)=-\infty$.

(ii $)|K(\theta)-K(\theta)| \leq M|\theta-\bar{\theta}|, \forall \theta, \bar{\theta} \leq \theta_{s}$.

For the new variable $\theta$ we obtained in fact the diffusive model

$$
\frac{\partial \theta}{\partial t}-\Delta \beta^{*}(\theta)+\frac{\partial K(\theta)}{\partial x_{3}}=f \quad \text { in } Q
$$

with the initial and boundary data.

The dimensionless diffusive model. We perform now the variable transformations

$$
\tilde{\theta}=\frac{\theta-\theta_{r}}{\theta_{s}-\theta_{r}}, \quad \tilde{t}=\frac{t}{t_{d}}, \quad \tilde{x}=\frac{x}{\lambda_{d}}, \quad \tilde{\beta}(\tilde{\theta})=\frac{\beta(\theta)}{\beta_{d}}, \quad \tilde{K}(\tilde{\theta})=\frac{K(\theta)-K_{r}}{K_{s}-K_{r}}
$$

in order to make the model dimensionless. Here $\lambda_{d}, t_{d}$ and $\beta_{d}$ are the characteristic length, time and diffusivity for the problem. All these lead to the dimensionless model

$$
\begin{gathered}
\frac{\partial \tilde{\theta}}{\partial t}-\Delta \tilde{\beta}^{*}(\tilde{\theta})+\frac{\partial \tilde{K}(\tilde{\theta})}{\partial \tilde{x}_{3}}=f \quad \text { in } Q, \\
\tilde{\theta}(\tilde{x}, 0)=\tilde{\theta}_{0}(\tilde{x}), \\
\left(\tilde{K}(\tilde{\theta}) i_{3}-\nabla \tilde{\beta}^{*}(\tilde{\theta})\right) \cdot v=\tilde{u}(\tilde{x}, \tilde{t}) \quad \text { a.e. on } \Sigma_{u}, \\
\left(\tilde{K}(\tilde{\theta}) i_{3}-\nabla \tilde{\beta}^{*}(\tilde{\theta})\right) \cdot v=\alpha \tilde{\beta}^{*}(\tilde{\theta})+\tilde{f}_{0} \quad \text { a.e. on } \Sigma_{\alpha} .
\end{gathered}
$$

The domain for $\tilde{\theta}$ is $\left[0, \tilde{\theta}_{s}\right)$ and in particular $\tilde{\theta}_{s}=1, \tilde{K}(0)=0$, and $\tilde{\beta}(0)=\tilde{\rho}>0$.

Up to now, the functions have been defined on the physical domain for the moisture, $\left(0, \tilde{\theta}_{s}\right)$, but for the application of the mathematical theory, they are extended to the left of the origin by continuity and in our paper we will denote still by $\beta, \tilde{\beta}^{*}(\tilde{\theta})$ and $K$ the following extensions

$$
\begin{gathered}
\tilde{\beta}(\tilde{\theta}):=\left\{\begin{array}{ll}
\rho, & \tilde{\theta} \leq 0 \\
\tilde{\beta}(\tilde{\theta}), & 0<\tilde{\theta}<\theta_{s} ;
\end{array} \quad \tilde{K}(\tilde{\theta}):= \begin{cases}0, & \tilde{\theta} \leq 0 \\
\tilde{K}(\tilde{\theta}), & 0<\tilde{\theta} \leq \theta_{s},\end{cases} \right. \\
\tilde{\beta}^{*}(\tilde{\theta}):= \begin{cases}\rho \tilde{\theta}, & \tilde{\theta}<0 \\
\int_{0}^{\tilde{\theta}} \tilde{\beta}(\xi) d \xi, & 0 \leq \tilde{\theta}<\tilde{\theta}_{s} \\
{\left[K_{s}^{*},+\infty\right),} & \tilde{\theta}_{s} \leq \tilde{\theta} .\end{cases}
\end{gathered}
$$


The conditions (i), (ii), ( $\left.\mathrm{ii}_{\mathrm{K}}\right)$ are preserved in the dimensionless model.

In the main part of the paper it is proved that under suitable conditions we have $\theta \in\left[0, \tilde{\theta}_{s}\right]$. This means that $\theta$ which is the real moisture belongs to $\left[\theta_{r}, \theta_{s}\right]$ and the corresponding pressure $h \in\left[h_{r},+\infty\right)$.

We have to specify that conditions $\left(\mathrm{a}_{1}\right)-\left(\mathrm{a}_{4}\right)$ which imply the conditions (i), (ii), (ii $\left.\mathrm{K}_{\mathrm{K}}\right)$ have been chosen such that to correspond to realistic hydraulic models used in soil sciences, that is, Broadbridge-White and van Genuchten. For example, we indicate that a limit case in the model of Broadbridge-White (see [6]) correspond to the functions

$$
\tilde{\beta}(\tilde{\theta})=\frac{1}{\sqrt{1-\tilde{\theta}}}, \quad \tilde{K}(\tilde{\theta})=\tilde{\theta}^{2}
$$

which satisfy all these conditions. This case describes a strongly nonlinear soil.

Therefore the model we deal with is (A.11) satisfying (i), (ii), and (ii $\mathrm{K}_{\mathrm{K}}$. For simplicity, in the main part of the paper we will not indicate the symbol " $\sim$ " but we will keep in mind that the results apply to the dimensionless form.

A.2. The smoother approximation. The proof of the existence of the free boundary requires some stronger regularity of the approximating solution that may be obtained using a smoother approximation $\beta_{\varepsilon}^{*}$ of class $C^{3}(\mathbb{R})$. So, we introduce the following function

$$
\beta_{\varepsilon}^{*}(\theta)= \begin{cases}\beta_{m} \theta, & \theta \leq \theta_{\mathrm{ext}} \\ \beta_{\mathrm{ext}}^{*}(\theta), & \theta_{\mathrm{ext}}<\theta \leq 0 \\ \beta^{*}(\theta), & 0<\theta \leq \theta_{s}-\varepsilon \\ \beta_{\text {int }}^{*}(\theta), & \theta_{s}-\varepsilon<\theta \leq \theta_{s} \\ \beta^{*}\left(\theta_{s}-\varepsilon\right)+\frac{K_{s}^{*}-\beta^{*}\left(\theta_{s}-\varepsilon\right)}{\varepsilon}\left[\theta-\left(\theta_{s}-\varepsilon\right)\right], & \theta>\theta_{s},\end{cases}
$$

where $\theta_{\text {ext }}$ is chosen such that $\left(\beta_{\text {ext }}^{*}\right)^{\prime}\left(\theta_{\text {ext }}\right) \stackrel{\text { def. }}{=} \beta_{m}>0$ with $\beta_{m}$ constant, independent on $\varepsilon$.

The function $\beta_{\mathrm{int}}^{*}$ and $\beta_{\mathrm{ext}}^{*}$ may read

$$
\begin{aligned}
& \beta_{\text {int }}^{*}(\theta)=\sum_{j=1}^{5} a_{j} \theta^{j}, \\
& \beta_{\text {int }}^{*}(\theta)=\sum_{j=1}^{5} b_{j} \theta^{j}
\end{aligned}
$$

with $a_{j}$ and $b_{j}$ chosen such that $\beta_{\text {int }}^{*} \in C^{3}\left[\theta_{s}-\varepsilon, \theta_{s}\right]$ and $\beta_{\mathrm{ext}}^{*} \in C^{3}\left[\theta_{\mathrm{ext}}, 0\right]$. 
The derivatives of $\beta_{\varepsilon}^{*}$ are

$$
\begin{aligned}
&\left(\beta_{\varepsilon}^{*}\right)^{\prime}\left(\theta_{\varepsilon}\right):=\beta_{\varepsilon}(\theta)= \begin{cases}\beta_{m}, & \theta \leq \theta_{\mathrm{ext}} \\
\left(\beta_{\mathrm{ext}}^{*}\right)^{\prime}(\theta), & \theta_{\mathrm{ext}}<\theta \leq 0 \\
\beta(\theta), & 0<\theta \leq \theta_{s}-\varepsilon \\
\left(\beta_{\mathrm{int}}^{*}\right)^{\prime}(\theta), & \theta_{s}-\varepsilon<\theta \leq \theta_{s} \\
K_{s}^{*}-\beta^{*}\left(\theta_{s}-\varepsilon\right) & \theta>\theta_{s},\end{cases} \\
& \beta_{\varepsilon}^{\prime}(\theta)= \begin{cases}0, & \theta \leq \theta_{\mathrm{ext}} \\
\left(\beta_{\mathrm{ext}}^{*}\right)^{\prime \prime}(\theta), & \theta_{\mathrm{ext}}<\theta \leq 0 \\
\beta^{\prime}(\theta), & 0<\theta \leq \theta_{s}-\varepsilon \\
\left(\beta_{\mathrm{int}}^{*}\right)^{\prime \prime}(\theta), & \theta_{s}-\varepsilon<\theta \leq \theta_{s} \\
0, & \theta>\theta_{s},\end{cases} \\
& \beta_{\varepsilon}^{\prime \prime}(\theta)= \begin{cases}0, & \theta \leq \theta_{\mathrm{ext}} \\
\left(\beta_{\mathrm{ext}}^{*}\right)^{\prime \prime \prime}(\theta), & \theta_{\mathrm{ext}}<\theta \leq 0 \\
\beta^{\prime \prime}(\theta), & 0<\theta \leq \theta_{s}-\varepsilon \\
\left(\beta_{\mathrm{int}}^{*}\right)^{\prime \prime \prime}(\theta), & \theta_{s}-\varepsilon<\theta \leq \theta_{s} \\
0, & \theta>\theta_{s},\end{cases}
\end{aligned}
$$

(we remind that we considered $\beta$ twice differentiable on $\left(0, \theta_{s}\right)$ ).

Therefore $\beta_{\varepsilon}, \beta_{\varepsilon}^{\prime}$ and $\beta_{\varepsilon}^{\prime \prime}$ are bounded on $\mathbb{R}$ and we have

$$
0<\beta_{m} \leq \beta_{\varepsilon}(\theta), \quad \forall \theta \in \mathbb{R}, \forall \varepsilon>0,
$$

so that relationship (ii) is satisfied with the constant $\beta_{m}$. Obviously all results proved for the approximation (1.16) remain true for the smoother approximation introduced before.

We also mention that, due to the convexity of the function $\beta$ on $\left(0, \theta_{s}\right)$, on the interval $\left[\theta_{s}-\varepsilon, \theta_{s}\right)$ we have the inequality

$$
\beta^{*}(\theta) \leq \beta^{*}\left(\theta_{s}-\varepsilon\right)+\frac{K_{s}^{*}-\beta^{*}\left(\theta_{s}-\varepsilon\right)}{\varepsilon}\left[\theta-\left(\theta_{s}-\varepsilon\right)\right],
$$

so that the function $\beta_{\text {int }}^{*}$ inserted in (A.14) has the property

$$
\beta^{*}(\theta) \leq \beta_{\text {int }}^{*}(\theta) \leq \beta^{*}\left(\theta_{s}-\varepsilon\right)+\frac{K_{s}^{*}-\beta^{*}\left(\theta_{s}-\varepsilon\right)}{\varepsilon}\left[\theta-\left(\theta_{s}-\varepsilon\right)\right],
$$

for $\theta \in\left[\theta_{s}-\varepsilon, \theta_{s}\right)$. Then it follows that

$$
\beta_{\varepsilon}^{*}(\theta) \geq \beta^{*}(\theta) \text { for } \theta \in\left[0, \theta_{s}\right)
$$


We still have

$$
\beta_{\varepsilon}^{*}(\theta) \geq K_{s}^{*} \quad \text { for } \theta \geq \theta_{s}
$$

\section{Acknowledgment}

This work was supported by the CNCSIS Grant Contract no. 33045/2004 financed by the Ministry of Education and Research.

\section{References}

[1] V. Barbu, Nonlinear Semigroups and Differential Equations in Banach Spaces, Noordhoff International Publishing, Leiden, 1976.

[2] - Partial Differential Equations and Boundary Value Problems, Mathematics and Its Applications, vol. 441, Kluwer Academic, Dordrecht, 1998.

[3] V. Barbu and G. Marinoschi, Existence for a time-dependent rainfall infiltration model with a blowing up diffusivity, Nonlinear Anal. Real World Appl. 5 (2004), no. 2, 231-245.

[4] I. Borsi, A. Farina, and A. Fasano, On the infiltration of rain water through the soil with runoff of the excess water, Nonlinear Anal. Real World Appl. 5 (2004), no. 5, 763-800.

[5] P. Broadbridge, Solution of a nonlinear absorption model of mixed saturated-unsaturated flow, Water Resources Research 26 (1988), no. 10, 2435-2443.

[6] P. Broadbridge and I. White, Constant rate rainfall infiltration: a versatile nonlinear model 1. Analytic solution, Water Resources Research 24 (1988), 145-154.

[7] P. Broadbrigde, J. H. Knight, and C. Rogers, Constant rate rainfall in a bounded profile: solutions of a nonlinear model, Soil Sci. Soc. Am. J. 52 (1988), 1526-1533.

[8] J.-L. Lions and E. Magenes, Non-Homogeneous Boundary Value Problems and Applications. Vol. I, Die Grundlehren der mathematischen Wissenschaften, vol. 181, Springer-Verlag, New York, 1972.

[9] G. Marinoschi, A free boundary problem describing the saturated-unsaturated flow in a porous medium, Abstr. Appl. Anal. 2004 (2004), no. 9, 729-755.

[10] M. T. van Genuchten, A closed-form equation for predicting the hydraulic conductivity of unsaturated soils, Soil Sci. Soc. Am. J. 44 (1980), 892-898.

Gabriela Marinoschi: Institute of Mathematical Statistics and Applied Mathematics, P.O. Box 1-24, Bucharest, Romania

E-mail address: gmarino@acad.ro 


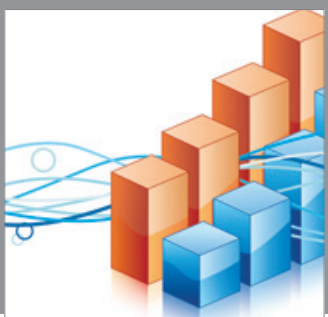

Advances in

Operations Research

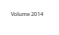

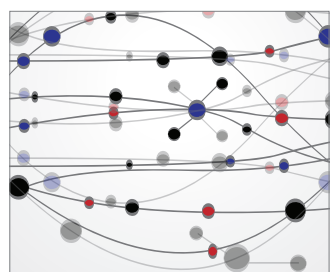

\section{The Scientific} World Journal
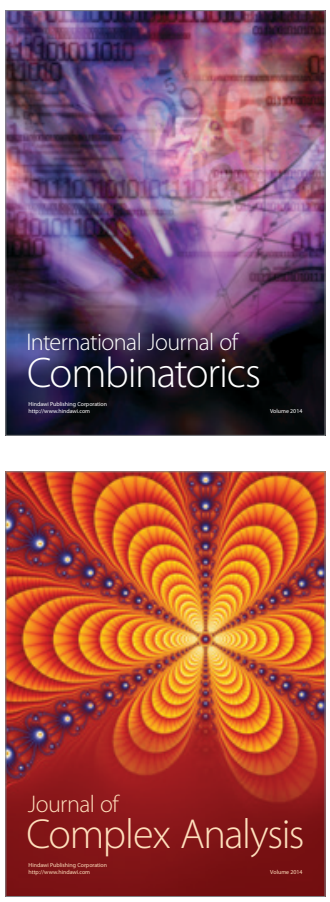

International Journal of

Mathematics and

Mathematical

Sciences
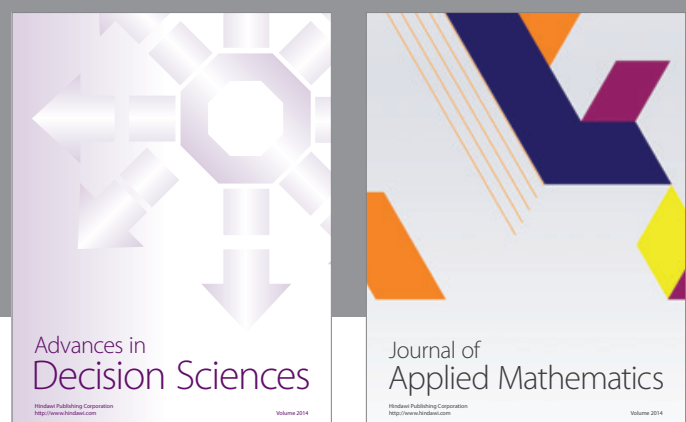

Journal of

Applied Mathematics
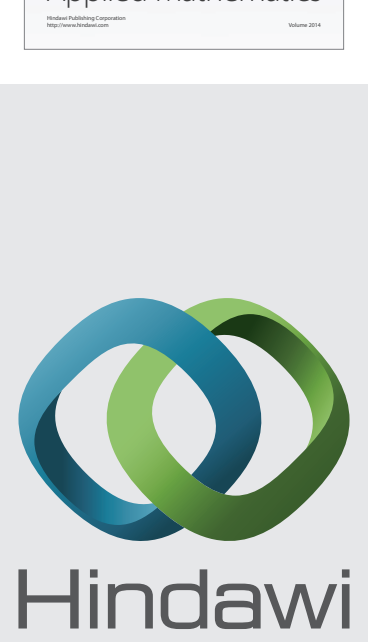

Submit your manuscripts at http://www.hindawi.com
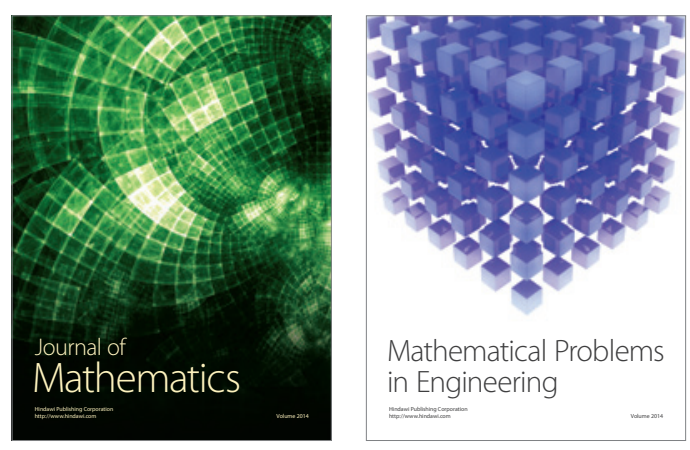

Mathematical Problems in Engineering
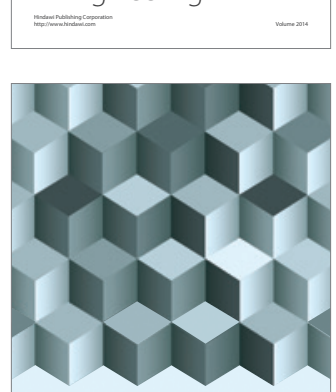

Journal of

Function Spaces
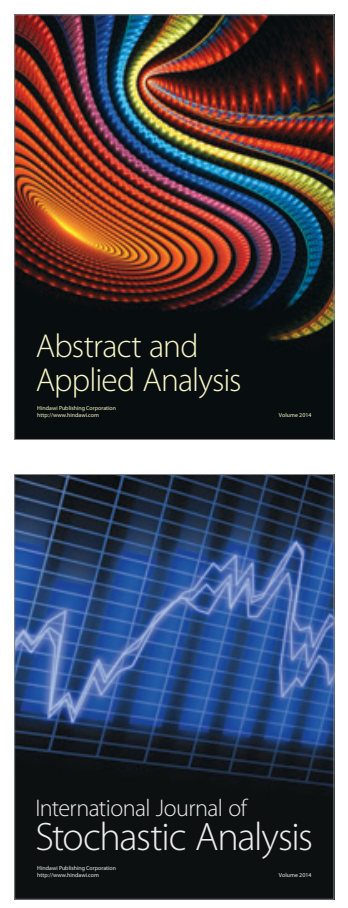

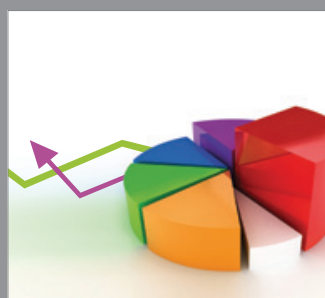

ournal of

Probability and Statistics

Promensencen
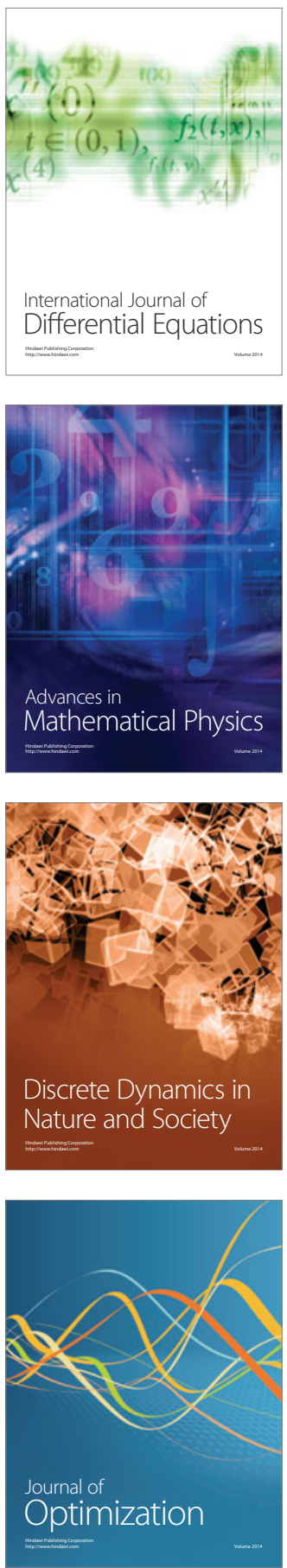